\title{
Quantitative comparison of Drosophila behavior annotations by human observers and a machine learning algorithm
}

Xubo Leng ${ }^{1,2 \dagger}$, Margot Wohl ${ }^{1,3 \dagger}$, Kenichi Ishii ${ }^{1}$, Pavan Nayak $^{1}$, Kenta Asahina ${ }^{1 *}$

1. Salk Institute for Biological Studies, La Jolla, CA, 92037, U.S.A.

2. Department of Electrical and Computer Engineering

3. Neuroscience Graduate Program, University of California, San Diego, La Jolla, CA, 92037, U.S.A.

* Correspondence: Kenta Asahina (kasahina@salk.edu)

${ }^{\dagger}$ Equal contributions 


\begin{abstract}
:
Automated quantification of behavior is increasingly prevalent in neuroscience research. Human judgments can influence machine-learning-based behavior classification at multiple steps in the process, for both supervised and unsupervised approaches. Such steps include the design of the algorithm for machine learning, the methods used for animal tracking, the choice of training images, and the benchmarking of classification outcomes. However, how these design choices contribute to the interpretation of automated behavioral classifications has not been extensively characterized. Here, we quantify the effects of experimenter choices on the outputs of automated classifiers of Drosophila social behaviors. Drosophila behaviors contain a considerable degree of variability, which was reflected in the confidence levels associated with both human and computer classifications. We found that a diversity of sex combinations and tracking features was important for robust performance of the automated classifiers. In particular, features concerning the relative position of flies contained useful information for training a machine-learning algorithm. These observations shed light on the importance of human influence on tracking algorithms, the selection of training images, and the quality of annotated sample images used to benchmark the performance of a classifier (the 'ground truth'). Evaluation of these factors is necessary for researchers to accurately interpret behavioral data quantified by a machine-learning algorithm and to further improve automated classifications.
\end{abstract}

\title{
Significance Statement:
}

Accurate quantification of animal behaviors is fundamental to neuroscience. Here, we quantitatively assess how human choices influence the performance of automated classifiers trained by a machine-learning algorithm. We found that human decisions about the computational tracking method, the training images, and the images used for performance evaluation impact both the classifier outputs and how human observers interpret the results. These factors are sometimes overlooked but are critical, especially because animal behavior is itself inherently variable. Automated quantification of animal behavior is becoming increasingly prevalent: our results provide a model for bridging the gap between traditional human annotations and computer-based annotations. Systematic assessment of human choices is important for developing behavior classifiers that perform robustly in a variety of experimental conditions. 


\section{INTRODUCTION}

Behavior is the ultimate output of the nervous system (1, 2). Accurate and quantitative measurements of behavior are vital for evaluating the effects of genetic, neuronal, pharmacological, or environmental perturbations on animals. Traditionally, measurement of behaviors performed by freely moving animals has relied on human observations. Recent advances in computational approaches have transformed this process by replacing human observations with automated computational processes that parameterize animal motions in a high-dimensional space. This information can be then used to classify specific actions through either a supervised or an unsupervised machine-learning algorithm (reviewed in 1,3-7). The obvious strengths of automated behavioral classification are the enormous data-processing capacity and the reproducibility of the results. A computer can apply the exact same criteria to every image file, in theory eliminating the variability that may exist within a human observer or among multiple observers. Moreover, an unsupervised machine-learning algorithm may identify new types of behavior that have escaped human attention.

However, computational measurements of behavior invite an inevitable question: how should we evaluate the performance of automated classification? The importance of this question is sometimes overlooked because it is rather trivial if the classification task is unambiguously binary. For example, a face recognition task answers a binary question ('is this person $\mathrm{A}$ or not A?'). Most behavioral classification tasks implicitly assume that the answer is likewise binary. However, comparison of the behavioral classifications among multiple observers reveals a considerable level of discrepancy (8-12) that challenges this assumption. This inter-observer variability is often used to promote the superiority of computer-based classification over human observation $(4,6)$. However, the performance of every machine-learning-based algorithm must be benchmarked against a "ground truth", which is the annotation by human observers (4). This means that the human selection of training and ground truth images inherently impact the performance of a computer-based classifier $(9,13)$; however, these factors have rarely been assessed systematically. The challenges become more significant as the scale of behavioral data continues to expand. The amount of actual behavioral data one observer can evaluate imposes limitations on the quality of the ground truth data used for performance evaluation and (especially for supervised learning) on the number of training images, which need to be sufficiently diverse to create a reasonably generalizable classifier $(9,14)$. It is therefore important to quantitatively assess the relationship between human factors and computational measurements under a variety of situations, especially for behaviors that are variably annotated by human observers. 
In this study, we aim to understand how factors controlled by humans, both during training and in the evaluation process, influence the performance of computer classifiers for animal behaviors (Fig. 1A). To this end, we first quantified the variability of human observations of three types of social behavior exhibited by pairs of fruit flies (Drosophila melanogaster) in multiple sex combinations. In parallel, we developed a series of supervised automated classifiers for these behaviors using a collection of training movies and then quantitatively compared the results of human and computer classification of another dataset. Our results show that the probability that a given behavior bout is detected as a particular behavior by the classifier correlates with the aggregated confidence levels of the human annotators. The performance of the classifiers improved as the diversity of the training files increased, mainly by reducing misclassification of types of behavior that were only present in a subset of movies. Each of the motion-related features curated by the creator of the tracking program assumed different levels of importance for each classifier; features concerning the relative position of the two flies helped improve the classification accuracy for social behaviors. These results suggest that the variability of human observations in fact reflects the variability inherent in animal behaviors, which can be quantified objectively by the confidence levels of well-trained automated classifiers. However, the noticeable impact of training file diversity on classifier performance indicates that it is vital for classifier creators to disclose the nature of the training files before applying the classifier to novel experimental paradigms.

\section{RESULTS}

\section{Consistency and variability of human classifications}

We first wished to quantify the variability in animal-behavior classifications made by human observers. To this end, we recorded interactions between a number of Drosophila pairs and had two trained observers independently annotate the behaviors. Both male and female flies show a variety of stereotypical actions in the context of social interactions. We focused on three types of actions: (1) unilateral wing extensions (henceforth referred to as wing extensions), which are an important part of male-type courtship behavior toward a female $(15,16),(2)$ lunges, which are a major component of attacks in inter-male aggressive behavior $(17,18)$, and (3) headbutts, which are a major component of attacks in inter-female aggressive behavior (19, 20). These three types of actions were chosen because they are frequently observed in specific combinations of the sexes and their motions are relatively unambiguous. We asked the observers to report not only the occurrence of these behaviors but also their subjective confidence level for 
each annotation, from 1 (least confident) to 3 (most confident). These graded annotations allowed us to quantify how conspicuous the given behavior appeared to human observers.

In all, movies of 30 pairs of flies with a total length of $\sim 534$ minutes were independently annotated by two human observers, who were assigned from a pool of four trained scientists (see Table 1 and Supplementary Data File for details). Each of the three behaviors was observed primarily for a specific combination of sexes, consistent with previous reports. Wing extensions were performed primarily by males toward females (a low number of male-to-male wing extensions were also observed; Fig. $1 \mathrm{~B}_{1}$ ). Lunges were performed exclusively among pairs of male flies (Fig. $1 \mathrm{C}_{1}$ ), while headbutts were performed predominantly among pairs of female flies (Fig. 1D 1 ). Although trained observers generally agreed on classification of behaviors, we found that a noticeable number of bouts were annotated by only one of the two observers. For wing extensions, $58.6 \%$ of the total annotated bouts received a combined confidence score of 4 6 from the two observers (Fig. $1 \mathrm{~B}_{2,4}$ ). However, $30.1 \%$ of the bouts were annotated by only one of the two observers (Fig. $1 \mathrm{~B}_{2,3}$ ). Likewise, $66.7 \%$ of the total annotated lunge bouts received a combined score of $4-6$ (Fig. $1 \mathrm{C}_{2,4}$ ), but $27.6 \%$ of the bouts were annotated by only one observer (Fig. $\left.1 \mathrm{C}_{2,3}\right)$. Lastly, $34.0 \%$ of the headbutt bouts were annotated by only one observer $\left(\right.$ Fig. $\left.1 \mathrm{D}_{3}\right)$. The more subtle nature of headbutt motions possibly accounts for the lower proportion of bouts that received a combined score of 4-6 (44.1\%) (Fig. 1D $\left.\mathrm{D}_{2,4}\right)$. These data suggest that even the "stereotypical" social behaviors of Drosophila contain a certain degree of perceived variability.

Lunges and headbutts are "ballistic" behaviors of short and relatively constant duration (median of $83 \mathrm{~ms}$ for lunges (Fig. 2A) and $67 \mathrm{~ms}$ for headbutts (Fig. 2B)). On the other hand, the duration of wing extensions can vary greatly (Fig. 2C, D). Interestingly, wing extensions of longer duration tended to be scored higher by human observers than wing extensions of shorter duration (Fig. 2E), suggesting that bout-based analysis of scores may underestimate the consistency of human annotations. Therefore, we analyzed the scores for wing extensions frame by frame. This analysis revealed that $70.6 \%$ of the frames received a combined score of $4-6$ and $23.4 \%$ of frames were annotated by only one observer (Fig. 3). These numbers suggest that consistency among observers is indeed higher at the frame level than at the bout level. We therefore perform both bout-based and frame-based analyses for wing extensions in the following sections. In general, we found that the difference between bout-based and frame-based analyses was quantitative rather than qualitative.

\section{Automated classifiers quantitatively reflect the confidence of human judgments}


Our observations above illuminate a noticeable degree of variability in the annotations of human observers. Similar variability has been reported when more than one person annotates the same movies of behaving nematodes $(8)$, flies $(9,10)$, and mice $(11,12)$. We wondered how this seemingly variable "ground truth" for animal behaviors would be reflected when benchmarking computer classification. To answer this question, we developed a set of well-trained automated classifiers for the three above-mentioned behaviors $(21,22)$ using the machine-learningbased platform JAABA (9). In each frame, parts of the fly body were labeled and parameterized using FlyTracker (23), which computes 13 basic feature values related to fly position and motion. The program then generates the first and second derivatives for each feature, creating 39 features in total that are subsequently utilized by JAABA. Compared with unannotated frames, frames annotated by human observers had distinct z-score distributions (Fig. 4A $A_{1}, B_{1}, C_{1}$ ) and variances (which likely result in distinct derivatives) (Fig. $\left.4 A_{2}, B_{2}, C_{2}\right)$. This suggests that FlyTracker features contain information that JAABA can use to differentiate annotated frames from non-behavior frames.

We aimed to develop JAABA classifiers that perform robustly. As training with a diverse set of movies is important for developing reliable JAABA classifiers $(9,24)$, we trained a wingextension classifier with 78,482 frames (1,308 seconds in total), a lunge classifier with 11,360 frames (189 seconds in total), and a headbutt classifier with 10,351 frames (173 seconds in total) (see Supplementary Table S1 and Supplementary Data File for a complete description of the training movies). Note that the fly pairs in the training frames were different from the fly pairs in the frames used for testing (i.e., the frames annotated by human observers). We also smoothed raw JAABA-detected bouts by eliminating bouts that were shorter than at least $98 \%$ of manually annotated bouts (Fig. 2A, B, D; see also Supplementary Data File), and by filling short gaps sandwiched by frames that received JAABA scores above the detection threshold value. These filters corrected for fragmentation of behavioral bouts that were sometimes generated by frame-based classification in JAABA (see Materials and Methods for details). We used recall (the ratio of human annotations detected by the classifier to all human annotations) and precision (the ratio of human annotations detected by the classifier to all classifier annotations) to evaluate the performance of the classifiers. The set of smoothing parameters that resulted in the optimal trade-off between recall and precision with a detection threshold of 0.1 was used for subsequent analyses unless otherwise noted (Fig. 5).

We then quantitatively compared the classification results from human observers and the JAABA classifiers by calculating recall and precision. We found that the JAABA classifiers reliably detected behavioral bouts that received high human confidence scores. For bouts that 
received a score of 4 or higher (Fig. $\left.1 \mathrm{~B}_{4}-\mathrm{D}_{4}\right)$, recall was $96.0 \%$ for the wing-extension classifier, $95.3 \%$ for the lunge classifier, and $87.9 \%$ for the headbutt classifier. All three JAABA classifiers had almost perfect recall for behavioral bouts that received a score of 6 (Fig. 6B, E, H).

As expected, the detection threshold was positively correlated with precision (Fig. 6A, D, G, Fig. 11A) and inversely correlated with recall (Fig. 6B, E, H, Fig. 11B) for all three behaviors. Interestingly, recall steadily decreased for all classifiers as the combined confidence scores dropped (Fig. 6B, E, H, Fig. 11B). For bouts that received scores of 3 or lower (Fig. 1B- $\left.\mathrm{B}_{4}\right)$, recall fell to $31.5 \%$ for wing extensions, $73.7 \%$ for lunges, and $64.3 \%$ for headbutts. Moreover, the median JAABA score tended to be lower for bouts with lower combined human scores (Fig. 6C, F, I, Fig. 11C); this accounts for the lower recall for bouts that received lower confidence scores from human observers. These observations indicate that benchmarking values (such as recall) can be quantitatively influenced by the ground truth annotation of human observers.

The apparent correlation between human confidence levels and JAABA scores is intriguing given that the JAABA classifiers were trained with binary labels ("true" or "false") instead of graded weights. JAABA scores of true and false for the training frames were largely, if not completely, separated (Fig. 6C, F, I, Fig. 11C), suggesting that the behaviors included in the training frames were mostly unambiguous. Recall would have no correlation with the combined confidence score if human confidence levels were randomly assigned subjective values that had no relationship with either the other observer's confidence levels or JAABA scores, which are objective "confidence" levels determined by the algorithm. Indeed, a permutation test confirmed that this correlation is highly unlikely to be generated by chance. When we randomized the human confidence scores to the bouts that JAABA detected in a size-matched manner, the probability of an uneven distribution of JAABA scores across human confidence scores was very small ( $p<0.01$ by Kruskal-Wallis test; Fig. 6J). The $p$-values from our experimental data were also many orders of magnitude smaller than the smallest $p$-values obtained with randomized samples (Fig. 6J). In addition, the 95\% confidence intervals of the expected average recall for permutated datasets were lower than the observed values for bouts with high combined confidence scores and higher for bouts with low scores (Fig. 6K). These results suggest that the confidence level of the human observers can be predicted to a certain extent by the JAABA classifiers.

We also noticed that false-positive bouts for all three behaviors had low JAABA confidence values (Fig. 6C, F, I, Fig. 11C), indicating that most false positives barely passed the detection threshold. To address whether these false positives stemmed from misclassification of 
certain types of motions, we manually inspected all the false-positive bouts (Tables 2-4). Interestingly, we found that a noticeable number of "false positives" appeared similar to true behaviors. These actions likely escaped the observers' attention. For the wing-extension classifier, $22 \%$ of "false positive" bouts appeared to be actual wing extensions (Table 2). For the lunge classifier, $25 \%$ appeared to be actual lunges or lunge-like motions that were not completed, and another $20 \%$ were lunge-like striking actions during high-intensity tussling (Table 3 ). For the headbutt classifier, $7 \%$ appeared to be actual headbutts, and another $20 \%$ were ambiguous "jerking" motions which were difficult to clearly distinguish from headbutts (Table 4). Excluding such incidents, the common false positives for the wing-extension classifier involved wing motions, such as grooming (Table 2). For the lunge and headbutt classifiers, short, quick motions (such as when a fly received a lunge or headbutt, or when a fly fell from the wall) were often misclassified (Table 3, 4).

These false positives can be at least partially explained by the types of feature-value deviations associated with a given behavior (Fig. 4). For instance, wing-extension frames annotated by human observers had high z-scores for maximum wing angle and high variance for minimum wing angle. Annotated lunge and headbutt frames had high z-scores and low variance for velocity. Together, these observations suggest that the source of the false positives was not necessarily random "noise". Although we made rigorous efforts to minimize common types of false positives during training, it proved difficult to eliminate them without sacrificing recall values (Fig. 6A, B, D, E, G, H).

Overall, these quantitative analyses suggest that the perceived variability in Drosophila behaviors is not solely due to subjective human artefacts, but is at least partially attributable to variability in the motion of the flies themselves. Deviations from "stereotyped" actions can be represented by lower confidence both in human observers and in automated behavioral classifiers. Inherent variability in animal behaviors that have historically been regarded as "stereotypical" is consistent with an emerging view that animal behaviors can be represented as a probability distribution in a continuous parameter space $(6,7,25)$.

\section{Diversity of training samples affects the robustness of automated classifiers}

Whether supervised or unsupervised, automated animal-behavior classifiers are developed with training samples, which are usually chosen by humans. These samples likely cover only a portion of the behavioral repertoire that a given animal species can exhibit. We next addressed how the choice of training movies affected the performance of our classifiers. 
A male fly performs wing extensions vigorously toward female flies, and to a lesser extent toward other males (see Fig. $1 \mathrm{~B}_{1}$ ). The recall of the wing-extension classifier trained only on movies of male-female pairs (48,992 frames) was comparable to the recall of the fully trained classifier (Fig. 7C, red). However, the precision was only 64.5\%, noticeably lower than that of the fully trained classifier (Fig. 7A, red). The lower precision was largely due to a high false-positive rate with male-male pair movies (Fig. 7B, red). Manual inspection revealed that a large number of false positives were fast flicking motions of wings that males often show when paired with another male. Addition of female-female (Fig. 7A, B, pink) and male-male (Fig. 7A, B, purple) training pairs steadily improved the precision. In contrast, the recall remained largely unaltered by the increase in the diversity of the training movies (Fig. 7C). (See also Fig. 11D-F for frame-based analyses.)

Lunges are performed predominantly among males. When the lunge classifier was trained only on movies of pairs of male flies (7,921 frames in total), its precision was $76.3 \%$, again lower than that of the fully trained classifier (Fig. 7D). False-positive bouts from this classifier largely occurred in movies of male-female pairs, while the false-positive rates in movies of male-male pairs remained largely the same (Fig. 7E). Manual inspection revealed that the majority of false positives in male-female pair movies came from misclassification of copulation attempts as lunges. The addition of male-female training pairs, which contained only negative training frames (1,927 frames, all labeled as "not lunge"), largely eliminated false positives in this condition (Fig 7D, purple), but also decreased the recall for bouts with relatively low combined human scores (1-4) (Fig. 7F, purple). The addition of female-female training pairs (395 frames) had little impact on precision (Fig. 7D, green), but the addition of both male-female and female-female training pairs improved the precision while limiting the decrease in recall (Fig. 7D-F, brown).

Lastly, for the headbutt classifier, both precision (Fig. 7G) and recall (Fig. 7I) increased as training movies of male-female pairs and male-male pairs were added. Unlike the wing-extension and lunge classifiers, the largest source of false positives was female-female pairs for all versions of the headbutt classifier trained with an intermediate diversity of training movies (Fig. $7 \mathrm{H}$ ). Nonetheless, the decrease in false positives when male-male and male-female training movies were added helped improve the precision (Fig. 7G, $\mathrm{H}$ ).

In the experiments above, more frames were added to the training set as the variety of training frames increased, raising the possibility that it was the increased number of training frames that led to the improvements in classifier performance. To address this, we trained classifiers on fewer training frames, while maintaining the training-frame diversity. We did this by 
randomly selecting training frames across all training movies, with the ratio of frames proportional to those used with the fully trained classifier (we call this process "downsampling" of the training frames). We found that a downsampling rate of $5 \%$ for the lunge classifier (Fig. 8D, E), and $25 \%$ for the wing-extension (Fig. 8A, B, Fig. 11G, E) and headbutt (Fig. 8G, H) classifiers, was sufficient to achieve precision comparable to the fully trained counterparts. Male-female pairs accounted for $62.3 \%$ of the entire training frames for the fully trained wing-extension classifier, male-male pairs accounted for $69.7 \%$ of the entire training frames for the lunge classifier, and female-female pairs accounted for $51.7 \%$ of the entire training frames for the headbutt classifier. When we adjusted the downsampling rate to these values, the precision of all classifiers was, predictably, similar to the precision of the fully trained classifiers (Fig. 7A, D, G). Moreover, their $95 \%$ confidence intervals were well above those of classifiers trained on a single type of fly pair. The results from the downsampled training indicate that the low precision of classifiers trained on limited types of training movies is not simply due to the reduction in the number of training frames relative to the fully trained classifiers.

For wing extension and lunges, classifiers trained on downsampled training frames and fully trained classifiers showed largely comparable recall (Fig. 7C, F, Fig. 11F). By contrast, the recall of the fully trained headbutt classifier was sometimes well above the $95 \%$ confidence intervals for recall of the downsampled headbutt classifiers (Fig. 7I). This could mean that the current fully trained headbutt classifier may be further improved by adding more training frames. It is also possible that the fully trained classifier might be over-fitted to the evaluation movies.

These results demonstrate that selecting a variety of training frames is critical for improving the robustness of the behavior classifiers, even if some of the movies do not contain the behavior of interest. Interestingly, classifiers trained solely with a specific type of movie appear comparable to the fully trained classifiers only when their performance was evaluated with the same type of movie (male-male pair movies for lunges, male-female pair movies for wing extensions, female-female pairs for headbutts). This suggests that a seemingly well-performing behavior classifier that is validated only for a specific combination of sexes may not perform well with other combinations.

\section{Robustness of classifiers when tracking information is incomplete}

In machine vision approaches that estimates an animal's pose $(13,23,26,27)$, animal posture is subdivided into a combination of parameters (features) that describe the position of each body segment (such as head, limbs, etc.). As previously mentioned, JAABA creates a series of rules that use feature and feature derivatives as inputs for its machine-learning algorithm (9). 
Importantly, these features and rules are defined by the creator of a program. We next examined whether a small number of features can contain sufficient information for JAABA-based behavior classifications, or whether a diversity of features and rules is collectively important for robust performance of a classifier.

Differences in the z-score distributions and variance of particular features between annotated and unannotated frames (Fig. 4) suggest that classifiers may utilize these information-rich features to distinguish behavior frames. Indeed, we found that each classifier used rules derived from each feature with different weights (Table 5). We re-trained classifiers after removing 1 or 3 of the most highly weighted features, and compared their precision and recall with those of the fully trained classifiers. The precision of these classifiers was noticeably lower than the precision of the fully trained classifiers. In particular, the bout-based precision of the wing-extension classifier without its most weighted feature, maximum wing angle, was only $44 \%$, and removal of the 3 most-weighted features further decreased the precision to $22 \%$ (Fig. 9A, dark purple; see also Fig. 11J for frame-based statistics). The precision of the lunge and headbutt classifiers showed qualitatively similar, but less exaggerated, trends (Fig. 9D, G, dark purple). By contrast, removal of the 3 least-weighted rules from the training process did not noticeably affect either precision (Fig. 9A, D, G, light gray bars) or recall (Fig. 9C, F, I, broken gray lines). Interestingly, classifiers lacking the most-weighted features retained the tendency to detect bouts with higher human confidence scores better than bouts with lower human confidence scores (Fig. 9C, F, I). Overall, these observations suggest that key features have a large impact on the reliable detection of a behavior.

We wondered whether these highly weighted features alone contain sufficient information to create reliable behavior classifiers. To test this possibility, we used only the three most highly weighted features when training the classifiers. Wing-extension classifiers trained this way performed surprisingly well, showing precision and recall very similar to the fully trained classifier (Fig. 9A-C, light purple). By contrast, the lunge and headbutt classifiers that were trained this way had $24 \%$ and $19 \%$ lower precision, respectively, than the corresponding fully trained classifiers (Fig. 9D-I, light purple). Together, these results show that information relevant for behavior classification can be distributed across many features, even though some features contribute more than others to the performance of a classifier. This underscores the value of using a variety of features when developing a reliable classifier.

JAABA rules further increase the diversity of information that can be useful for behavior classification. A list of the most-weighted rules for each classifier (Supplementary Table S2) indicates that all three classifiers distribute weights to rules derived from a relatively large number 
of features, suggesting that aggregates of diverse rules can collectively encode information relevant for behavioral classification. Indeed, when we disabled usage of the 3, 5, or 10 mostweighted rules for fully trained classifiers, both precision (Fig. 10A, B, D, E, G, H) and recall (Fig. 10C, F, I) degraded only marginally. Upon removal of these rules, other rules that derived from corresponding features often took over as the most-weighted rules (Supplementary Table S2), suggesting that rules derived from the same feature may contain redundant information for reliable behavioral classification.

Under natural conditions, social behaviors are expressed largely toward other animals and, to a certain extent, are defined in relation to the other animals. This raises the possibility that the accurate classification of a social behavior may require information about the relative positions of the participating animals, such as the distance between the two individuals, the orientation angle, and so on. We tested this possibility by training the classifier on the same set of training frames but without using the features that concern the relative position of the two flies ("relative features"). Among the 13 basic features, 9 features were defined solely by the autonomous properties of a single fly, whereas the remaining 4 were relative features (Fig. 4) (23). At least one relative feature was among the 3 most-weighted features for all three fully trained classifiers (Table 5). We found that both the precision and recall of the classifiers trained without relative features decreased modestly. The decrease in bout-based precision was $4.3 \%$ for the wing-extension classifier, $8.5 \%$ for the lunge classifier, and $7.5 \%$ for the headbutt classifier (Fig. 9A, D, G, Fig. 11J, dark blue), and was due to an increase in false-positive rates across movie types (Fig. 9B, E, H, Fig. 11K, dark blue). Bout-based recall across all human score categories was $-1.8 \%$ for the wing-extension classifier, and $-2.7 \%$ for the lunge and headbutt classifiers (Fig. 9C, F, I, dark blue). These results indicate that classifier accuracy can benefit from information about the relative position of the flies.

\section{DISCUSSION}

Measurement of animal behavior is integral to behavioral neuroscience. With technological advances in recording and movement tracking, many types of automated approaches have been applied to quantify animal behaviors (reviewed in $6,7,28$ ). However, even automated approaches involve human choices and judgments, from the design of the algorithm to the choice of training and benchmarking samples. Relatively few systematic efforts have been made to quantitatively evaluate the impact of human factors at each step, or to reconcile automated results with results obtained from traditional human observation. Here, we systematically compared the annotation of Drosophila social behaviors by a group of human observers and by 
JAABA-based automated classifiers under a variety of perturbations. Our results lead to three important conclusions. First, the variability of human annotations is correlated with the "confidence" levels of automated classifiers, suggesting that the animal behaviors themselves are the source of at least part of the variability. Second, the use of diverse training samples is crucial to ensure robust performance of automated classifiers. Lastly, information about the relative position of experimental animals can improve the accuracy of automated classifiers.

Some classification tasks can assume an unambiguous ground truth. For instance, the goal of a human face-recognition task is binary: does a given face belong to the target person or not? Behavioral classification can involve more nuanced situations because a degree of variability may exist even in what is traditionally regarded as "stereotypical behavior" (7). Animals can abort ongoing behavior before completion (29), or can show variability in the execution of a learning-dependent motor program during $(30,31)$ or even after $(32)$ training. We found that human observers gave different levels of confidence in their classification of Drosophila social behaviors. It is possible to argue that such inter-observer variability would disappear with perfectly trained observers. However, inconsistencies among human observers have been repeatedly noted across the behavior classification of multiple species (8-12). In the absence of a clear definition of a "perfectly trained observer", these variable human classifications need to be accepted as the "ground truth" when evaluating the performance of automated classifiers (4). It is therefore practically important to assess how the results of automated behavior classification should be benchmarked against a variable "ground truth". We found that the level of human confidence and JAABA confidence values correlated when behavioral bouts were aggregated. Although we tried to avoid using frames with perceived ambiguous behaviors for both positive and negative training), it is possible that some mislabeling of training frames could have caused ambiguous classification (9). However, because of the diversity of training frames used in this study, we favor an alternative possibility: that the behaviors performed by pairs of flies contain inherent variability. This conclusion implies that it may not be possible to define the ground truth in a binary fashion. If a behavior - even one originally defined by human observers - is distributed across confidence-level space, the boundaries defining the behavior are ultimately decided by human judgment, much like a critical $p$-value in statistics. Additionally, our observations reinforce the idea that "subjective" human confidence levels can reflect statistically defined discriminability (33), and argue against the notion that variability in human judgments is simply randomly generated noise. We may be forced to reckon with the inherent variability of animal behaviors as technology to monitor animal movements continues to improve. 
At the same time, it is noteworthy that "high-confidence" behavioral bouts are indeed clearly identified by both humans and automated classifiers, which underscores the reported stereotypy of some animal behaviors $(15,18)$. As an operational variable, nomenclature for stereotypical behaviors remains useful for characterizing the nature of behavior and, importantly, necessary to bridge human observations from previous studies with data obtained through automated systems. This is particularly relevant for unsupervised behavioral classification, because the resulting clusters of "behaviors" are named by a human observer $(34,35)$, sometimes without clear descriptions of the nature of the variability within the given cluster. For specific purposes, eliminating low-confidence behavioral bouts from analyses may be justified $(9,36)$. However, these bouts may not be equivalent to non-behavior frames. Instead, they may have biological consequences in the context of social interactions. One possible solution is to weigh behavioral bouts according to the confidence value each bout receives. A scoring approach has been taken for the quantitative evaluation of other biological phenomena, such as dye-based feeding amount $(37,38)$ or aberrations of neuronal morphologies $(39,40)$.

Diverse training images are important for developing a robust automated classifier because machine-learning algorithms generally assume that the parameter space is the same for the training samples and the testing samples $(9,28)$. However, how automated classifiers perform on types of movies that are not a part of the training images has not previously been quantitatively analyzed. As expected, we found that classifiers trained with movies that contained only one combination of the sexes had lower precision than the fully trained classifier. Interestingly, the precision and recall of these partially trained classifiers were comparable to the values of the fully trained classifiers when the training and evaluation movies were of the same type. For instance, the wing-extension classifier trained only on male-female pair movies performed as well as the fully trained wing extension classifier when evaluating male-female pair movies only. While these results are not surprising in light of the nature of machine-learning algorithms, they illuminate a source of misinterpretation that may go unnoticed. An experimenter may be satisfied with the performance of an automated classifier on the basis of limited evaluation examples and overlook the errors that the classifier may commit for types of movies that the experimenter did not include in either the training or evaluation processes. This situation is analogous to gender- and race-dependent classification bias by a face-recognition algorithm that was not trained on a diverse dataset of faces. The bias became apparent only after diverse faces were used for benchmarking (14).

The degree of image diversity needed for optimal performance of a given automated classifier inherently depends on the type of movies used for the experiment. Since we do not 
know a priori the parameter space of fly behavior under different genetic and environmental manipulations, one possible interim solution is to fully disclose the nature of the training and evaluation images so that a new user can be aware of possible limitations for generalization. In the long run, a common depository of behavioral movies taken from a variety of genotypes under diverse conditions, much like a large dataset for annotating objects (41) and human motions (42), may help in the development of a universally applicable behavioral classifier.

Accurate tracking of animals is essential for successful automated classification (28). The tracking program we used in this study segments fly body parts in two-dimensional images (23). This means that the tracking becomes inaccurate when the fly changes body orientation along the z-axis. In fact, a certain portion of false positives in all three classifiers were detected when a fly was on the wall, which violates the tracking program's assumptions about the appearance of the body. One solution is to force an animal to pose in largely expected ways in a spatially restrictive arena $(25,26,43)$, but it is possible that such environmental constraints can put artificial limitations on animal behaviors. Deploying multiple cameras with different angles (44), or a depth-sensitive camera $(45,46)$, enables the three-dimensional posture of animals to be visualized and provides more comprehensive information about animal posture. Each of our classifiers used different features with different weights (for instance, the wing-extension classifier relied on the maximum wing angle, for an obvious reason), suggesting that certain features are critical for accurate detection of behaviors (28). At the same time, our finding that even features and rules with relatively low weights contribute to improving the performance of classifiers is noteworthy. One interesting question is how multiple pose-estimation packages $(13,27)$ will perform for automated behavior classification and how animal behavioral classification systems built on pose estimation compare against pixel-based behavioral classifications that do not assume specific animal postures $(25,46)$.

Interestingly, we also found that the automated classifiers for social behaviors give considerable weight to information related to the relative positions of the animals involved. Elimination of these relative features modestly but noticeably deteriorates the performance of classifiers, suggesting that these features are valuable for accurate behavior classification. Consistent with our observation, a recent report shows that relative features distribute distinctively between different behaviors and states during male courtship behavior to females (47). For a machinevision system that tracks animals, calculation of relative features can become computationally expensive as the number of tracked animals increases. As interest in detecting and quantifying social behavior grows, it may become necessary to identify a set of relative features that (1) has high discriminability (47) and (2) a low computational burden (28). 
Beyond standardizing behavioral quantification in a reproducible manner, automated behavioral classification methods have the potential to reveal motion patterns and behavioral dynamics that may escape human attention. It is important to note that it is ultimately human intuition and judgment that allows interpretation of results from often multidimensional automated classification. Just like carefully curated expert annotations of select genomic regions greatly facilitated automated annotation of the entire genome, detailed annotations of example animal movies through synthesis of multiple experts' observations will be foundational to ensuring that automated behavioral classification is as informative and objective as possible.

\section{ACKNOWLEDGEMENTS}

We thank Dr. Pietro Perona and Eyrun Arna Eyjolfsdottir for developing and improving FlyTracker during the course of this study, Isabella Stelter for assisting with the evaluation of JAABA classifiers, Dr. Terrence Sejnowski and Jorge Aldana for providing us with computational resources, infrastructure, and technical support, and Drs. Eiman Azim, Daniel Butler, and Saket Navlakha for helpful discussions. Stocks obtained from the Bloomington Drosophila Stock Center (NIH P40OD018537) were used in this study. K.I. was supported by the Naito Foundation and the Japan Society for the Promotion of Science Postdoctoral Fellowship Abroad. M.W. was supported by the Mary K. Chapman Foundation and the Rose Hills Foundation. This work was supported by NIH NIGMS R35 GM119844 to K.A. K.A. is a recipient of the Helen McLoraine Development Chair of Neurobiology at the Salk Institute.

\section{AUTHOR CONTRIBUTIONS}

K.A. conceived the project. M.W., K.I., P.N., and K.A. annotated fly behaviors. M.W. generated MATLAB scripts for processing Fly Tracker and JAABA outputs. X.L. and M.W. generated MATLAB scripts for quantitative comparisons between human annotated bouts and JAABA bouts. M.W. and X.L. also generated MATLAB scripts for visualizing the analysis results. K.A. and X.L. interpreted the results. K.A. wrote the manuscript, which X.L., M.W., and K.I. edited. 


\section{MATERIALS AND METHODS}

\section{Experimental Animals}

The complete genotypes of Drosophila are listed in Table 1 and Supplementary Table S1. TkGAL41 (48), P1 a split GAL4 (49) (R15A01-p65AD:Zp (in attP40) (RRID:BDSC_68837); R71G01Zp:GAL4DBD (in attP2) (RRID:BDSC_69507)), Otd-nls:FLP (48), UAS-dTRPA1 (50), and 20XUAS-FRT-dSTOP-FRT-CsChrimson:mVenus (in attP2) (51) are gifts from David Anderson (California Institute of Technology). 20XUAS-CsChrimson:tdTomato (in VK00022), 20XUASFRT-myr:TopHAT2-FRT-CsChrimson:tdTomato (in VK00022 or in attP2) were created by Barret Pfeiffer in the lab of Gerald Rubin (HHMI Janelia Research Campus) and kindly shared by David Anderson. NP2631 (52) is a gift from Daisuke Yamamoto (Tohoku University). fru ${ }^{M}$, (RRID:BDSC_66874) and fruF (RRID:BDSC_66873) (53) are gifts from Barry Dickson (HHMI Janelia Research Campus). $d s x^{F L P}$ (54) is a gift from Stephen Goodwin (University of Oxford). TRH-GAL4 (RRID:BDSC_ 38389) is a gift from Matthew Kayser (University of Pennsylvania). NPF-GAL4 (RRID:BDSC_25682), TRH-GAL4 (RRID:BDSC_38388), 20XUAS-

CsChrimson:mVenus (in attP40) (RRID:BDSC_55135), and fru ${ }^{4-40}$ (RRID:BDSC_66692) were obtained from Bloomington Drosophila Resource Center in the University of Indiana.

All flies were collected as virgins and were maintained at $25^{\circ} \mathrm{C}, 60 \%$ relative humidity. Virgin males and females were reared in a vial with standard Drosophila cornmeal media for 6 days, except flies that carry $d s x^{F L P}$ (see Supplementary Data File for details), which were reared for 14 days. For optogenetic experiments, the tester flies were reared on food containing 0.2 mM all-trans retinal (MilliporeSigma, Cat\#R2500, 20 mM stock solution prepared in 95\% ethanol), and vials were covered with aluminum foil to shield light. Mated wild-type (Canton-S) females were prepared by allowing wild-type males to mate with 4-day old virgin females for 2 days. Flies were transferred to vials containing fresh food media every 3 days until the day of the experiment.

\section{Behavioral Assays}

Behavioral assays were conducted as described elsewhere (21), although the stimulation paradigms differed between movies. Thermogenetic neuronal manipulations were conducted as described in (48). Briefly, vials that contain testing flies were incubated in a water bath at $28^{\circ} \mathrm{C}$ for 20 minutes, and were transferred to a behavioral arena. Recording was started 5 minutes after the transfer to allow flies to acclimate. All movies were recorded in .AVI format at 60fps.

As explained in more detail in the following section, recorded fly pairs were separated into "training" pairs and "evaluation" pairs. Training pairs were used for developing JAABA- 
based behavior-specific classifiers, while evaluation pairs were used for both behavior annotations by human observers and for the evaluation of JAABA classifier performances in comparison to human annotations. A fly pair used for one purpose was never used for another. The separation of training and evaluation pairs is necessary to prevent "over-fitting" of the learning algorithm.

See Supplementary Table S1 for details of experimental conditions for each movie.

\section{Tracking of Flies}

Acquired movies were first processed by the FlyTracker package version 1.0.5 (23) (http://www.vision.caltech.edu/Tools/FlyTracker/), which runs on MATLAB (The Mathworks, Inc.). The regions of interest were manually defined as circles or rectangles that correspond to the chamber of each arena. Foreground and body thresholds were adjusted for each movie for optimal segmentation of body and wing across at least 30 random frames. Note that segmentation is prone to error when two flies are in proximity or overlapping, or a fly is climbing a wall. Some of these cases were discussed in Result section. All tracking parameters can be found in the 'calibration.mat' file associated with each movie.

The identities of flies were confirmed for the following cases: 1) a male-female pair, in which case the sex was identified by the body size and morphology of the posterior end of the abdomen, (2) a male-male pair in which a wing of one of the flies was clipped for identification. The switching of two flies ('identity swap') was manually corrected using the 'Identity correction' function of the "visualizer" program in the FlyTracker package.

\section{Human Behavioral Annotations}

Each observer annotated wing extensions, lunges, and headbutts using the "Behavior annotation" function of the "visualizer" program. An observer first determined start and end frames for a given behavioral bout. Then, an observer used the "Certainty" section to specify how confident the annotation for each bout was with three levels: 'maybe' (1), 'probably' (2), and 'definitely' (3). This annotation process created a behavior-specific data structure in which a human confidence score of 0 (no label), 1, 2, or 3 is given to each frame for each fly of every evaluation pair. A single confidence score is assigned to a bout. In rare cases in which the fly of interest performed a behavior continuously with a changing degree of perceived certainty, an observer split the given bout into multiple "bouts", and gave different values of confidence to each bout. While all observers used all three confidence levels for all three annotated behaviors, the relative frequency 
of use of these confidence levels was discretionary to each observer. Prior to annotating, observers watched select frames of training pair movies together to form a general consensus on target behaviors. Each observer completed annotation independent of each other.

A movie of an evaluation pair was annotated by two observers. To create combined human annotated bouts, Bout-based combined human annotation was created by merging overlapping annotations via the union operation. For any given bout, start or end frames annotated by the two observers may be shifted, due to subjective judgement regarding which frame the start or end of the behavior is called. For wing extensions, we sometimes found cases in which two annotators segmented bouts differently, and a bout defined by one observer was divided into 2 or more bouts by another observer. These cases were considered as separate bouts, even though the union operation creates one combined human annotation in such cases. We declared that a bout is annotated by two observers if the bouts from the two observers overlapped for one frame $(17 \mathrm{~ms})$ or more. For a bout that was annotated by one observer, start and end frames recorded by the observer become the combined human annotation.

The confidence scores given by the two observers to a bout were summed to produce the human combined score for this bout (resulting in a 1-to-6 confidence scale). When more than one bout annotated by the first observer corresponded to one bout annotated by the second observer, we recorded the human combined score for each of the separate bouts. As is detailed below, the highest confidence score among the multiple bouts was used as a representative score of the first observer when a single human combined score is needed for this type of situation.

Frame-based combined human annotation was created by calculating the sum of confidence scores by the two observers for every frame.

\section{Training of Automated Classifiers}

Frame-by-frame classifiers for wing extensions, lunges, and headbutts were created using the machine learning algorithm JAABA (9). As stated above, we only used training pairs for classifier development. For each movie, a JAABA folder was created after the identity correction was complete. An .xml file that allows the FlyTracker output to be read in the JAABA platform ('featureConfigEyrun.xml') is available in Supplementary Data File.

Details of all training frames for or against each behavior for all training pair movies are available in Supplementary Data File. These frames were accumulated through iterative improvements of classifiers. First, a few dozen bouts of clear behaviors and a similar number of obvious non-behavior frames were labeled as the true behavior and "none", respectively. After 
initial training, training pairs were classified, and obvious sources of false positives were marked as "none", while a behavior of interest which did not receive high confidence values by the interim classifier was labeled as a "true" behavior. These processes were repeated until we did not observe noticeable improvements of performance, at which point the classifier was considered fully trained. This definition is operational for this study, and it is not meant that the "fully trained" classifier was expected to perform under any experimental conditions or for any genotypes.

Classifiers trained by a subset of training pairs were generated by first removing training frame labels from the fully trained classifier for the given behavior. The classifier was then retrained anew using only the frames from the specific type of training pairs. Classifiers trained without specific features or rules were generated from the fully trained classifiers in the following steps, except for classifiers trained without relative features. First, features or rules were removed by unchecking the target features or rules in the "Select Features" function in JAABA. The classifier was then re-trained using the same training frames and settings, and saved as a new classifier.

A classifier that did not use relative features was created in the following steps. First, a new JAABA project, in which relative features were removed a priori using the "Choose perframe features" function at the opening window, was created. Movies that contain all training pairs along with all training labels were then imported from the corresponding fully trained classifier using the "Import Exp and Labels from Jab" function. The classifier was then re-trained, and saved as a new classifier.

For downsampling of the training frames, the number of training frames for each fly, for each label ('behavior' or 'non-behavior'), was reduced according to the downsampling ratio by randomly choosing the training frames. The precise downsampling rate slightly deviates from the labeled rate in figure panels, as downsampling seldom generates integer frame numbers, and at least one frame was chosen from each fly for each label regardless of the downsampling rate. Ten independent downsampling and training iterations were applied for each downsampling rate to calculate the average and $95 \%$ confidence intervals of precision, false positive rates, and recall. See Supplementary Table S3 for details of classifiers with downsampled training frames.

\section{Comparison of human annotation and JAABA classification}

JAABA classifies a behavior by returning a confidence score for each frame. For bout-based evaluation, we first defined a JAABA bout as a series of continuous frames that has a JAABA 
confidence score higher than the threshold value. Since this is a frame-by-frame classification, fragmentation of a seemingly single behavioral bout can happen when a bout is relatively short (often the case for lunges or headbutts; see Fig. 2A, B), or at the edge of a JAABA-defined bout. As was discussed in the main text along with Fig. 4, we applied a maximum gap filling filter and minimum bout length filter to smooth the fragmented JAABA bouts. We first converted the nonJAABA bout frames (with a JAABA score below the threshold) that are sandwiched by frames with a JAABA score higher than the threshold to JAABA bout frames, while keeping the JAABA score intact. Then, we searched for bouts that had a duration of or above the minimum bout length of choice. After this smoothing process, we recorded the start and end frames of each of the corrected JAABA bouts. The average JAABA score for a given JAABA bout was calculated as (sum of frame-by-frame JAABA score within the JAABA bout)/(total number of JAABA bout frames).

To compare human annotation and JAABA classification on a per-bout-basis, we matched JAABA bouts to combined human annotated bouts using the following procedure. A combined human annotated bout was declared "matched" if a JAABA bout overlapped with the combined human annotated bout for one or more frames (over $17 \mathrm{~ms}$ ). Such a human annotated bout is called a true positive bout. In reality, human annotated bouts and JAABA bouts do not always match one-to-one. For bout-based evaluation, a human annotated bout was used as a reference. Specifically, when one human annotated bout overlapped with multiple JAABA bouts, the JAABA bouts were collectively counted as 1 matched bout. When more than one human annotated bout overlapped with a single JAABA bout, the number of human annotated bouts was the number of matched bouts. To put it the other way, a true positive bout is a category based upon a combined human annotated bout, and not on a JAABA bout.

A false negative bout was defined as a human annotated bout that did not match to any JAABA bout. A false positive bout was defined as a JAABA bout that did not match to any combined human annotation. For a false negative bout, we created a virtual JAABA bout, with length equal to the length of the human annotation intersection. The average JAABA score for these false negative bouts were calculated for the duration of this virtual JAABA bout. For a false positive bout, we created a virtual "human annotated" bout with its human combined score of 0 . The average JAABA score for combined human annotated bouts was first calculated using all frames in matching JAABA bouts, which was then associated to a corresponding combined human annotated bout. We chose to do this because (1) it is difficult to split a combined human annotation when an annotation by one observer overlapped with multiple annotations by another observer, and (2) this operation is consistent with our calculation of the average JAABA 
score for false positive bouts. In the case (1), we used the highest combined human score as the representative value for the bout. This means that the total number of bouts present in these figure panels are slightly less than the total number of the combined human annotated bouts used elsewhere (such as for calculation of precision and recall). When a single combined human annotated bout was matched by multiple JAABA bouts, the average JAABA score was calculated as an average of all the matched JAABA bouts. When multiple combined human annotated bouts were matched by a single JAABA bout, the single average JAABA score was assigned to each of the matched combined human annotated bouts.

A precision rate represents the ratio of true positive bouts among all positive bouts (true or false), and was calculated as follows:

(number of true positive bouts)/[(number of true positive bouts) + (number of false positive bouts)]

A recall rate represents the ratio of true positive bouts among the total human annotated bouts, and was calculated as follows:

(number of true positive bouts)/[(number of true positive bouts) + (number of false negative bouts)]

For frame-based comparison, each frame is declared "matched" if the frame has simultaneously 1) a JAABA score that is above threshold, and 2) a combined human score of 1 or higher. A frame that has a combined human score of 1 or higher, but a JAABA score below threshold is declared false negative, and a frame that has an above-threshold JAABA score but no human annotation is declared a false positive. Precision and recall rates were calculated in the same manner as for bout-based comparison.

\section{Generation of shuffled dataset}

To address whether the perceived correlation between human combined scores and the average JAABA scores could be observed by chance, we created a series of shuffled data sets from our experimental data set by randomizing a confidence score between 1 and 6 for each human annotated bout, at the same time preserving the percentage of each score category as in the original data. The false positive bouts were kept as false positives. We created 50 such shuffled data sets to evaluate the parameter distributions for statistical analyses.

\section{Statistical analysis}


The details of results of all statistical tests are shown in Supplementary Data File. Kruskal-Wallis one-way ANOVA test was used to address whether distributions of average JAABA scores for human annotated bouts are correlated with combined human scores. When the p-value was below the critical value of 0.01 , the pairwise Mann-Whitney U-test was used to ask whether the median of average JAABA scores for bouts with neighboring combined human scores was significantly different. For this test, we combined both true positive bouts and false negative bouts that had the given combined human scores. Since a total of 5 tests were performed for each behavior, the critical p-value was adjusted with Bonferroni correction to 0.05/5 $=0.01$.

For comparison of shuffled data sets and the experimental data set, the null hypothesis was that there was no correlation between human combined scores and the average JAABA score for a given human annotated bout. If the null hypothesis was correct, we would expect that 1) the observed distribution of average JAABA scores for human annotated bouts would be within a variation reasonably expected by the randomized data set, and 2) the observed recall rate for each human combined score would fall within the range of fluctuations reasonably expected from the randomized data sets. To test the first possibility, we performed the KruskalWallis test for each of 50 shuffled data sets. To test the second possibility, we calculated "recall" rates for bouts that belong to each combined human score in each shuffled data set. We then calculated the $95 \%$ confidence intervals of the "recall" rates for shuffled data sets, for each combined human score, and asked whether the observed recall rate was within the interval.

In Fig. 6C, 2F, 2I, and Fig. 11C, a violin plot (created by Bastian Bechtold; https://github.com/bastibe/Violinplot-Matlab) was used to represent relative abundance of the number of JAABA bouts or frames that have each of the 7 human combined scores (including false positives, which has a score of 0 ). For a combined human annotation that had multiple overlaps, the highest human combined score among those separate bouts was taken as the representative human combined score. Violins were created separately for true positives and false negatives. The width of a violins represents the kernel density estimate of the JAABA score statistics for all bouts or frames within the violin. Subsequently, the width of violins for a given behavior was scaled by the ratio relative to the category with the largest number of bouts or frames. Violins for training bouts or frames were created separately, and their width were adjusted according to the relative abundance between positive and negative training bouts or frames.

Ninety-five percent confidence intervals for shuffled datasets (Fig. 6J, K) and classifiers with downsampled training frames (Fig. 7, 8, 11D-I) were calculated using $t$ distribution as follows: 


$$
m \pm t_{0.025}(\mathrm{n}-1) \times s / \sqrt{ } \mathrm{n}
$$

Where $\mathrm{n}$ is the sample number (10 in this case), $m$ is the sample mean, $s$ is the standard error, and $t_{0.025}(\mathrm{n}-1)$ is the upper $0.05 / 2=0.025$ critical value for the $t$-distribution with $\mathrm{n}-1$ degrees of freedom ( 9 in this case). 


\section{REFERENCE}

1. Gomez-Marin A, Paton JJ, Kampff AR, Costa RM, \& Mainen ZF (2014) Big behavioral data: psychology, ethology and the foundations of neuroscience. Nat Neurosci 17(11):1455-1462.

2. Krakauer JW, Ghazanfar AA, Gomez-Marin A, Maclver MA, \& Poeppel D (2017) Neuroscience Needs Behavior: Correcting a Reductionist Bias. Neuron 93(3):480-490.

3. Dell Al, et al. (2014) Automated image-based tracking and its application in ecology. Trends Ecol Evol 29(7):417-428.

4. Anderson DJ \& Perona P (2014) Toward a science of computational ethology. Neuron 84(1):1831.

5. Egnor SE \& Branson K (2016) Computational Analysis of Behavior. Annu Rev Neurosci 39:217236.

6. $\quad$ Berman GJ (2018) Measuring behavior across scales. BMC Biol 16(1):23.

7. Datta SR, Anderson DJ, Branson K, Perona P, \& Leifer A (2019) Computational Neuroethology: A Call to Action. Neuron 104(1):11-24.

8. Szigeti B, Stone T, \& Webb B (2016) Inconsistencies in C. elegans behavioural annotation. BioRxiv.

9. Kabra M, Robie AA, Rivera-Alba M, Branson S, \& Branson K (2013) JAABA: interactive machine learning for automatic annotation of animal behavior. Nat Methods 10(1):64-67.

10. Kain J, et al. (2013) Leg-tracking and automated behavioural classification in Drosophila. Nat Commun 4:1910.

11. Garcia VA, Crispim Junior CF, \& Marino-Neto J (2010) Assessment of observers' stability and reliability - a tool for evaluation of intra- and inter-concordance in animal behavioral recordings. Conf Proc IEEE Eng Med Biol Soc 2010:6603-6606.

12. Burgos-Artizzu XP, Dollár P, Lin D, Anderson DJ, \& Perona P (2012) Social behavior recognition in continuous video. in 2012 IEEE Conference on Computer Vision and Pattern Recognition (IEEE, Providence, RI), pp 1322-1329.

13. Mathis A, et al. (2018) DeepLabCut: markerless pose estimation of user-defined body parts with deep learning. Nat Neurosci 21(9):1281-1289.

14. Buolamwini J \& Gebru T (2018) Gender Shades: Intersectional Accuracy Disparities in Commercial Gender Classification. in Proceedings of the 1st Conference on Fairness, Accountability and Transparency, eds Sorelle AF \& Christo W (PMLR, Proceedings of Machine Learning Research), pp 77--91.

15. Hall JC (1994) The mating of a fly. Science 264(5166):1702-1714.

16. Murthy M (2010) Unraveling the auditory system of Drosophila. Curr Opin Neurobiol 20(3):281287.

17. Jacobs ME (1960) Influence of light on mating of Drosophila melanogaster. Ecology 41(1):182188.

18. Chen S, Lee AY, Bowens NM, Huber R, \& Kravitz EA (2002) Fighting fruit flies: a model system for the study of aggression. Proc Natl Acad Sci U S A 99(8):5664-5668.

19. Ueda A \& Kidokoro Y (2002) Aggressive behaviours of female Drosophila melanogaster are influenced by their social experience and food resources. Physiol Entomol 27(1):21-28.

20. Nilsen SP, Chan YB, Huber R, \& Kravitz EA (2004) Gender-selective patterns of aggressive behavior in Drosophila melanogaster. Proc Natl Acad Sci U S A 101(33):12342-12347.

21. Ishii K, Wohl M, DeSouza A, \& Asahina K (2020) Sex-determining genes distinctly regulate courtship capability and target preference via sexually dimorphic neurons. Elife 9 .

22. Wohl M, Ishii K, \& Asahina K (2020) Layered roles of fruitless isoforms in specification and function of male aggression-promoting neurons in Drosophila. Elife 9.

23. Eyjolfsdottir E, et al. (2014) Detecting Social Actions of Fruit Flies. Lect Notes Comput Sc 8690:772-787.

24. Robie AA, et al. (2017) Mapping the Neural Substrates of Behavior. Cell 170(2):393-406 e328.

25. Berman GJ, Choi DM, Bialek W, \& Shaevitz JW (2014) Mapping the stereotyped behaviour of freely moving fruit flies. J $R$ Soc Interface 11(99).

26. Branson K, Robie AA, Bender J, Perona P, \& Dickinson MH (2009) High-throughput ethomics in large groups of Drosophila. Nat Methods 6(6):451-457. 
27. Pereira TD, et al. (2019) Fast animal pose estimation using deep neural networks. Nat Methods 16(1):117-125.

28. Robie AA, Seagraves KM, Egnor SE, \& Branson K (2017) Machine vision methods for analyzing social interactions. J Exp Biol 220(Pt 1):25-34.

29. Briggman KL, Abarbanel HD, \& Kristan WB, Jr. (2005) Optical imaging of neuronal populations during decision-making. Science 307(5711):896-901.

30. Jin X \& Costa RM (2010) Start/stop signals emerge in nigrostriatal circuits during sequence learning. Nature 466(7305):457-462.

31. Olveczky BP, Andalman AS, \& Fee MS (2005) Vocal experimentation in the juvenile songbird requires a basal ganglia circuit. PLoS Biol 3(5):e153.

32. Sossinka R \& Bohner J (1980) Song Types in the Zebra Finch Poephila-Guttata-Castanotis. Z Tierpsychol 53(2):123-132.

33. Sanders JI, Hangya B, \& Kepecs A (2016) Signatures of a Statistical Computation in the Human Sense of Confidence. Neuron 90(3):499-506.

34. Berman GJ, Bialek W, \& Shaevitz JW (2016) Predictability and hierarchy in Drosophila behavior. Proc Natl Acad Sci U S A 113(42):11943-11948.

35. Markowitz JE, et al. (2018) The Striatum Organizes 3D Behavior via Moment-to-Moment Action Selection. Cell 174(1):44-58 e17.

36. Fujiwara T, Cruz TL, Bohnslav JP, \& Chiappe ME (2017) A faithful internal representation of walking movements in the Drosophila visual system. Nat Neurosci 20(1):72-81.

37. Al-Anzi B, et al. (2010) The leucokinin pathway and its neurons regulate meal size in Drosophila. Curr Biol 20(11):969-978.

38. Jourjine N, Mullaney BC, Mann K, \& Scott K (2016) Coupled Sensing of Hunger and Thirst Signals Balances Sugar and Water Consumption. Cell 166(4):855-866.

39. Marin EC, Watts RJ, Tanaka NK, Ito K, \& Luo L (2005) Developmentally programmed remodeling of the Drosophila olfactory circuit. Development 132(4):725-737.

40. Hattori D, et al. (2009) Robust discrimination between self and non-self neurites requires thousands of Dscam1 isoforms. Nature 461(7264):644-648.

41. Deng J, et al. (2009) ImageNet: A large-scale hierarchical image database. 2009 IEEE Conference on Computer Vision and Pattern Recognition, (IEEE), pp 248-255.

42. Monfort M, et al. (2019) Moments in Time Dataset: one million videos for event understanding. in arXiv (arXiv:1801.03150)

43. Simon JC \& Dickinson MH (2010) A new chamber for studying the behavior of Drosophila. PLoS One 5(1):e8793.

44. Azim E, Jiang J, Alstermark B, \& Jessell TM (2014) Skilled reaching relies on a V2a propriospinal internal copy circuit. Nature 508(7496):357-363.

45. Hong W, et al. (2015) Automated measurement of mouse social behaviors using depth sensing, video tracking, and machine learning. Proc Natl Acad Sci U S A 112(38):E5351-5360.

46. Wiltschko AB, et al. (2015) Mapping Sub-Second Structure in Mouse Behavior. Neuron 88(6):1121-1135.

47. Calhoun AJ, Pillow JW, \& Murthy M (2019) Unsupervised identification of the internal states that shape natural behavior. Nat Neurosci 22(12):2040-2049.

48. Asahina $\mathrm{K}$, et al. (2014) Tachykinin-expressing neurons control male-specific aggressive arousal in Drosophila. Cell 156(1-2):221-235.

49. Hoopfer ED, Jung Y, Inagaki HK, Rubin GM, \& Anderson DJ (2015) P1 interneurons promote a persistent internal state that enhances inter-male aggression in Drosophila. Elife 4:e11346.

50. Hamada FN, et al. (2008) An internal thermal sensor controlling temperature preference in Drosophila. Nature 454(7201):217-220.

51. Shirangi TR, Wong JW, Truman DL, \& Stern DL (2016) Doublesex Regulates the Connectivity of a Neural Circuit Controlling Drosophila Male Courtship Song. Dev Cell 37(6):533-544

52. Yu JY, Kanai MI, Demir E, Jefferis GS, \& Dickson BJ (2010) Cellular organization of the neural circuit that drives Drosophila courtship behavior. Curr Biol 20(18):1602-1614.

53. Demir E \& Dickson BJ (2005) fruitless splicing specifies male courtship behavior in Drosophila. Cell 121(5):785-794. 
bioRxiv preprint doi: https://doi.org/10.1101/2020.06.16.153130; this version posted June 16, 2020. The copyright holder for this preprint (which was not certified by peer review) is the author/funder, who has granted bioRxiv a license to display the preprint in perpetuity. It is made available under aCC-BY-NC-ND 4.0 International license.

54. Rezaval C, Nojima T, Neville MC, Lin AC, \& Goodwin SF (2014) Sexually dimorphic octopaminergic neurons modulate female postmating behaviors in Drosophila. Curr Biol 24(7):725-730. 
bioRxiv preprint doi: https://doi.org/10.1101/2020.06.16.153130; this version posted June 16, 2020. The copyright holder for this preprint (which was not certified by peer review) is the author/funder, who has granted bioRxiv a license to display the preprint in perpetuity. It is made available under aCC-BY-NC-ND 4.0 International license.

Figure 1

A

Recording movies

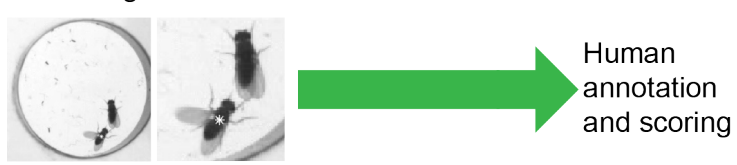

Supervised training $\checkmark$ (JAABA)

Tracking (FlyTracker)
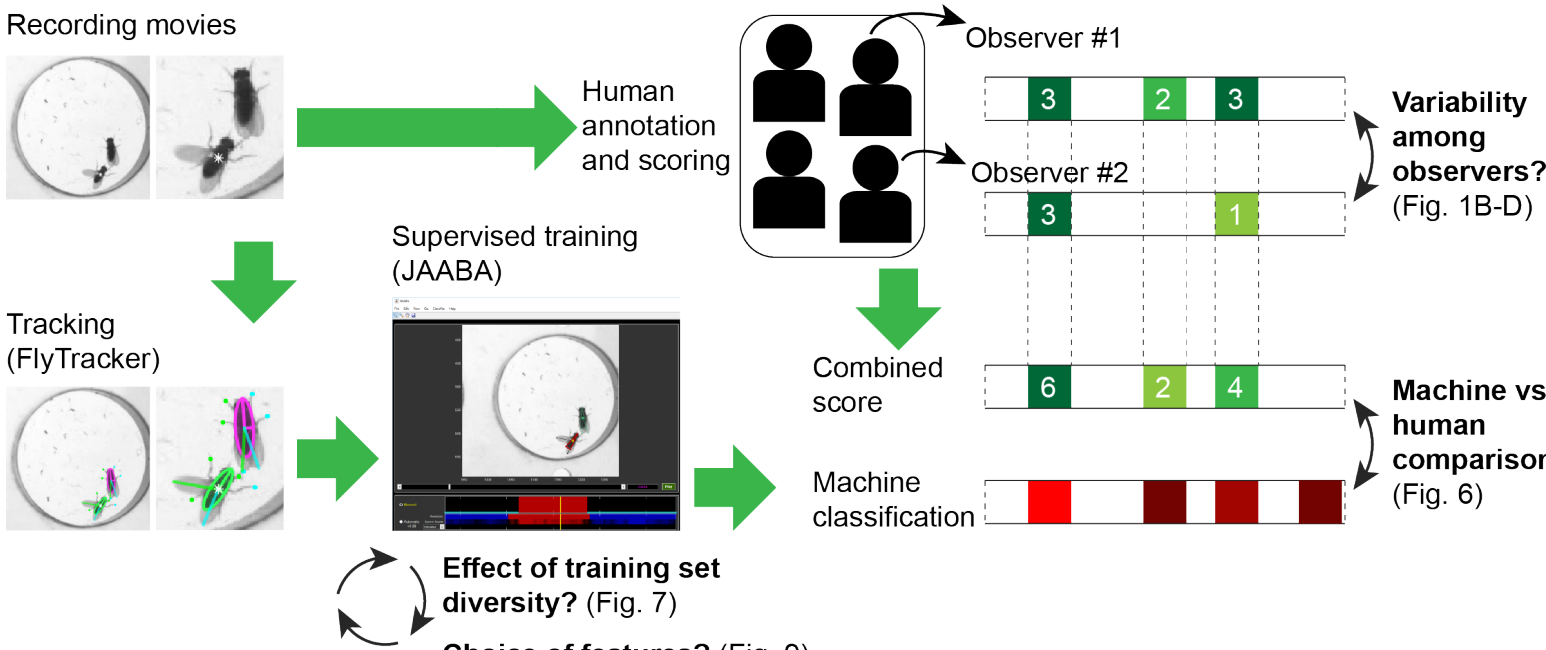

Effect of training set diversity? (Fig. 7)

Choice of features? (Fig. 9)

Wing extension

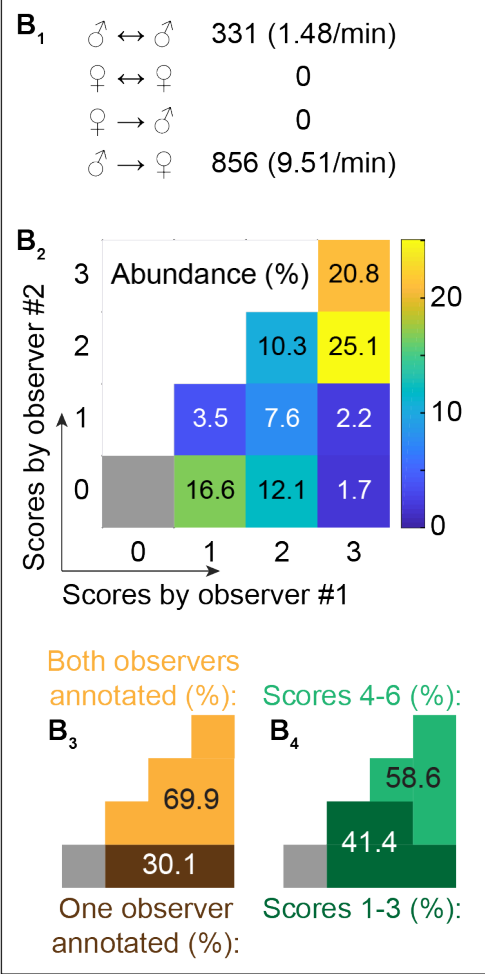

Lunge

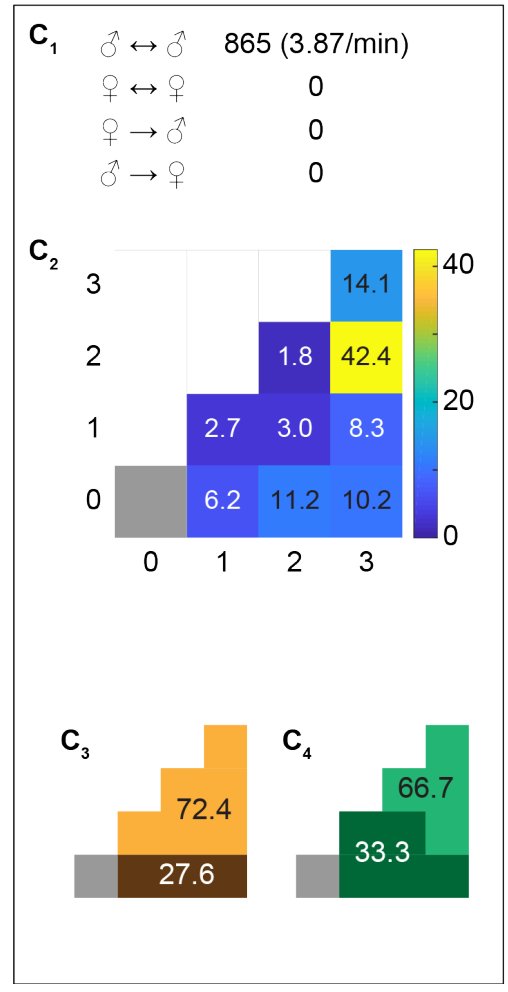

Headbutt

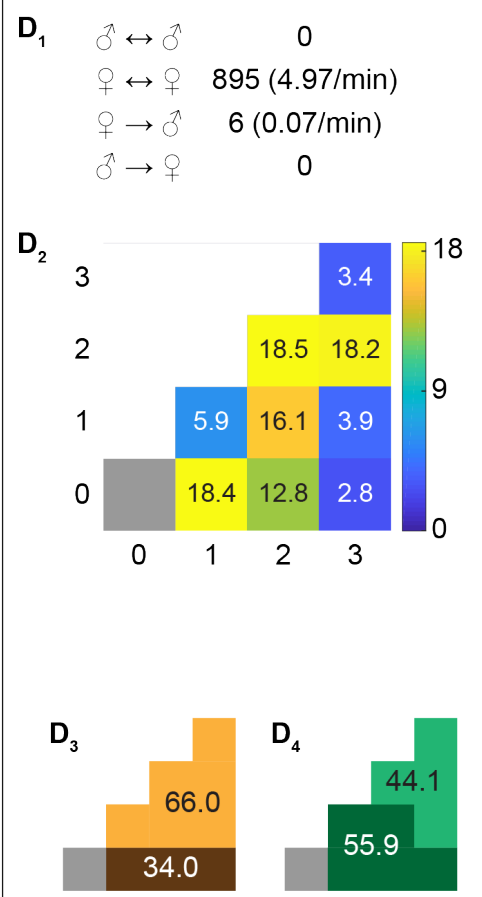


Figure 1: Variability of human annotation of Drosophila social behaviors.

A. Schematic of workflow and evaluations performed in this study. Movies of a pair of Drosophila adults were annotated both by human observers and by machine-learning-based automated classifiers. Inter-observer variability was quantified (B-D) and the performance of human and machine annotations were subsequently compared (Fig. 6). The effects of the diversity of training movies (Fig. 7) and features (Fig. 9) were also quantified. B-D. Summary of human annotations for wing-extension (B), lunge (C), and headbutt (D) behaviors. The total number of annotated behavioral bouts and frequency are categorized according to interaction type in $\mathbf{B}_{1}-\mathbf{D}_{1}$. The distributions of human score combinations are shown in 4-by-4 grids with pseudocolor representing relative abundance (scale bars on the right of each grid) $\left(\mathbf{B}_{2}-\mathbf{D}_{2}\right)$, and are also broken down according to whether bouts were counted by one or two observers $\left(B_{3}-D_{3}\right)$ and by combined score $\left(\mathbf{B}_{4}-\mathbf{D}_{4}\right)$. 
Figure 2
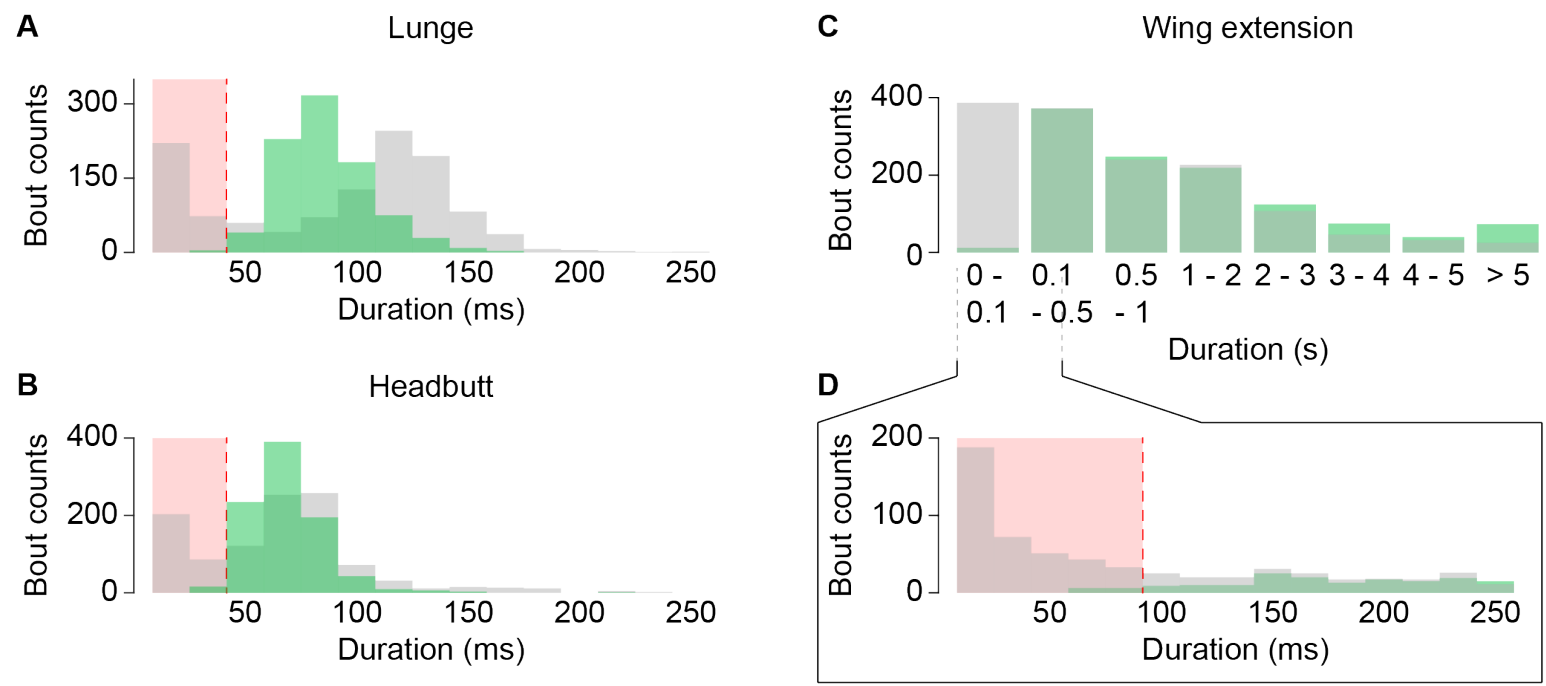

Manually annotated bouts

Bouts detected by classifiers

Eliminated by minimum bout length filter

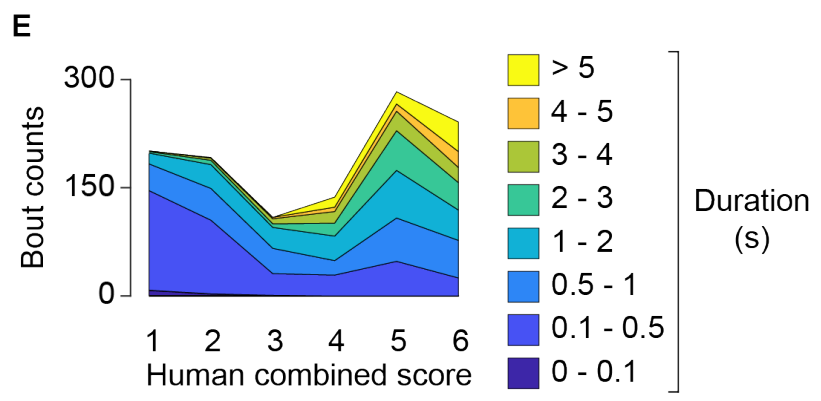

\section{Figure 2. Bout duration distributions for each behavior.}

A-D. Histograms of bout duration for lunge (A), headbutt (B), and wing extension (C, D). Green is the distribution of human annotated bouts, and gray is the distribution of JAABA bouts. For lunges and headbutts, bout duration was binned for every $17 \mathrm{~ms}$ (duration of one frame). For $\mathbf{C}$, bins are indicated below the plot. $\mathbf{D}$ is the magnified histogram for durations between 0 and 250 ms. Red shades show the durations that are eliminated by the minimum bout length filter (see text and Fig. 5). E. Distribution of wing extension bout durations according to human combined score. Bins are color-coded as shown on the right. 


\section{Figure 3}

Wing extension (frame-based)

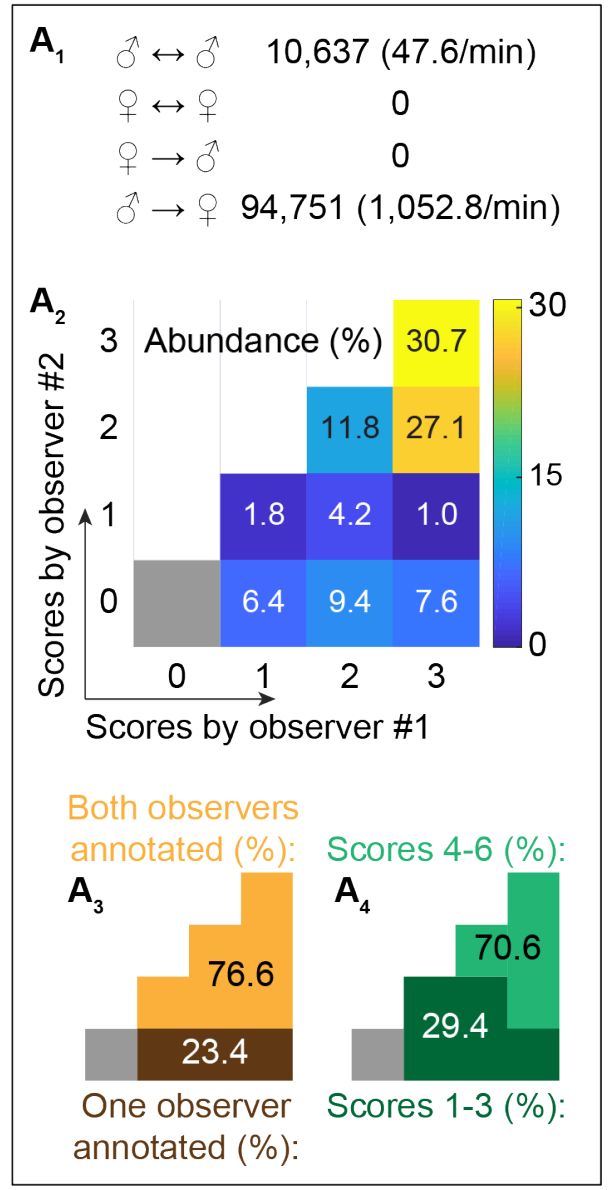

Figure 3. Frame-based statistics of human-annotated wing extensions.

Frame-based summary of human annotations for wing extension $\left(\mathbf{A}_{1}\right)$, categorized according to interaction types. Distribution of human score combinations are shown in 4-by-4 grid with pseudocolor $\left(\mathbf{A}_{2}\right)$ that represent relative abundance (scale bars on the right of the grid), and the breakdown according to whether frames were counted by one or two observers $\left(A_{3}\right)$ and combined scores $\left(\mathbf{A}_{4}\right)$ are also shown. Source data is identical to that used in Fig. 1B. 
Figure 4

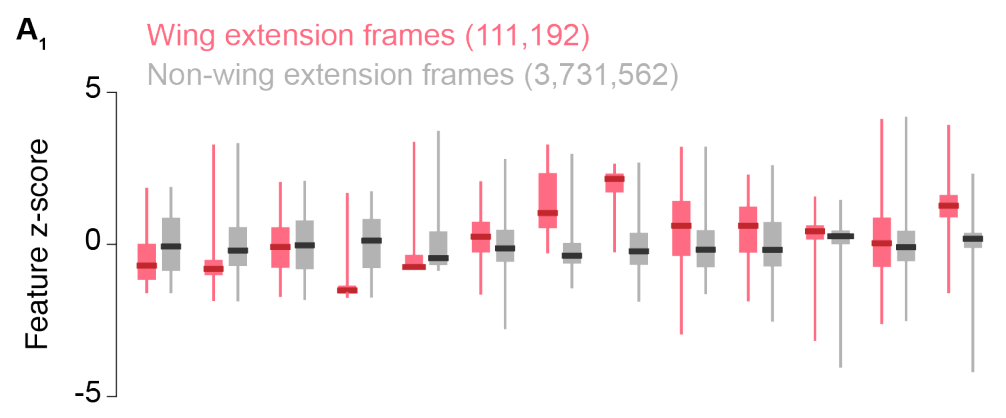

$A_{2}$

B $\quad$ Lunge frames $(4,618)$

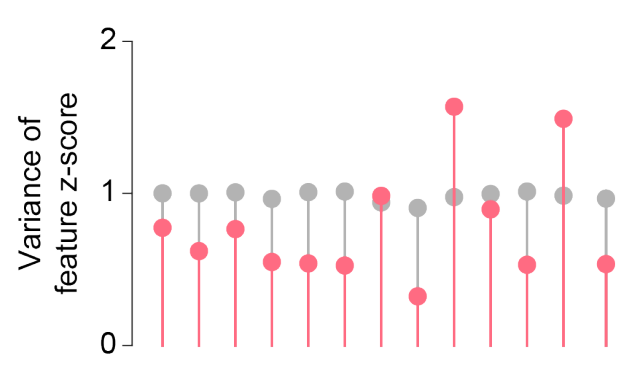

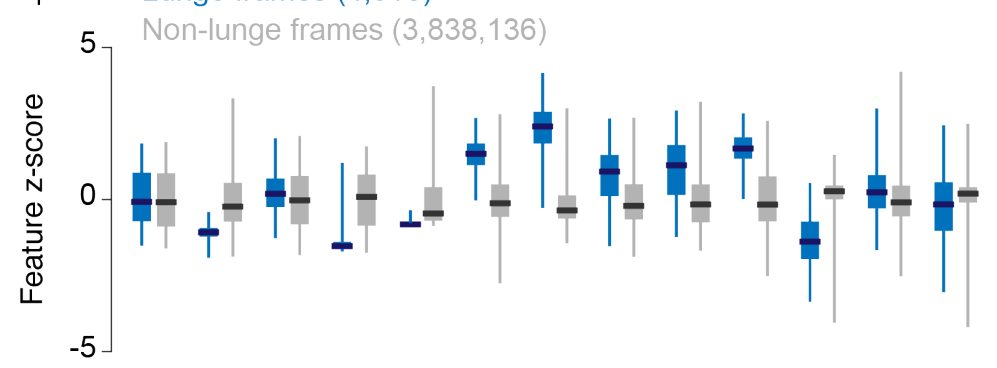

$\mathrm{B}_{2}$

$\mathbf{C}_{1} \quad$ Headbutt frames $(3,692)$

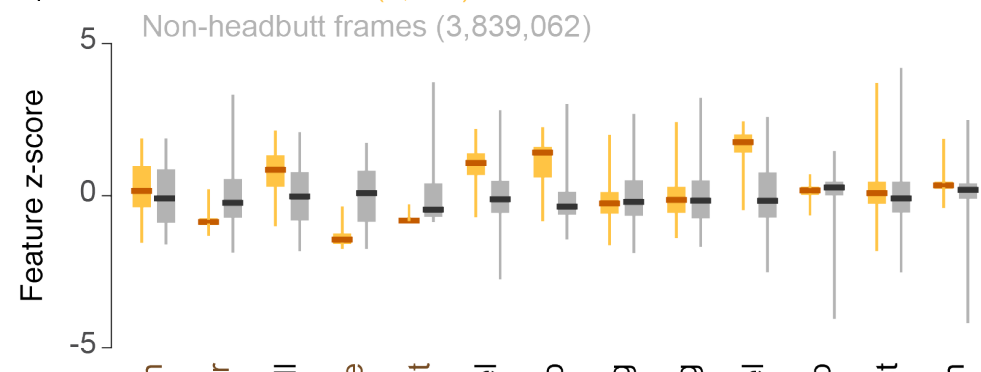

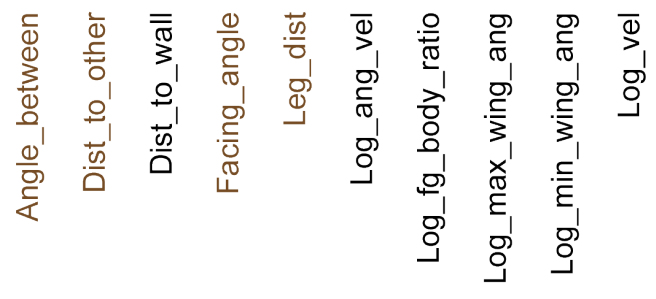

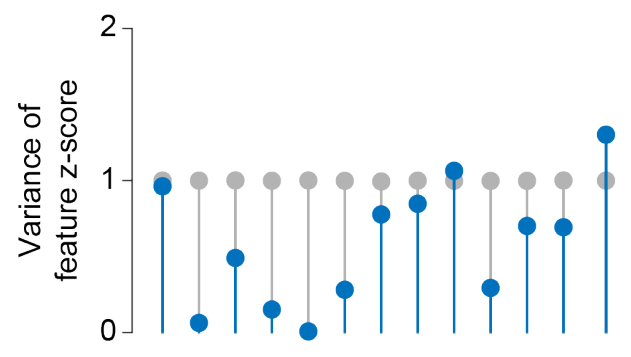

$\mathrm{C}_{2}$

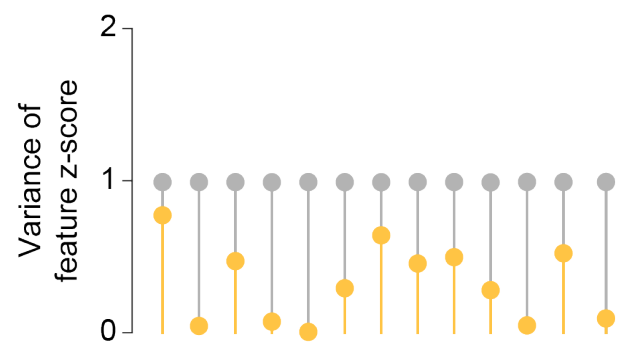

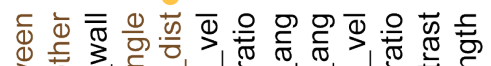

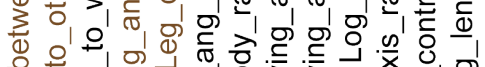

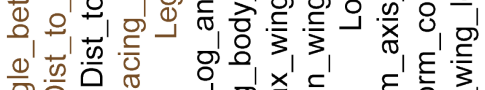

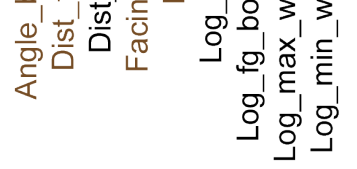

Figure 4. Frame-based z-score distributions and their variances according to behavior labels.

Distribution of frame-based feature z-scores $\left(\mathbf{A}_{\mathbf{1}}, \mathbf{B}_{\mathbf{1}}, \mathbf{C}_{\mathbf{1}}\right)$ and variances $\left(\mathbf{A}_{\mathbf{2}}, \mathbf{B}_{\mathbf{2}}, \mathbf{C}_{\mathbf{2}}\right)$ according to human annotations for wing extension (A), lunge (B), and headbutt (C). Z-score distributions are plotted in boxplot, where a thick bar represents median, a box represents 25 and 75 percentiles, and whiskers represents 0.5 and 99.5 percentiles. Features calculated from relative positions of the 2 flies (relative features) are shown in brown. 
Figure 5

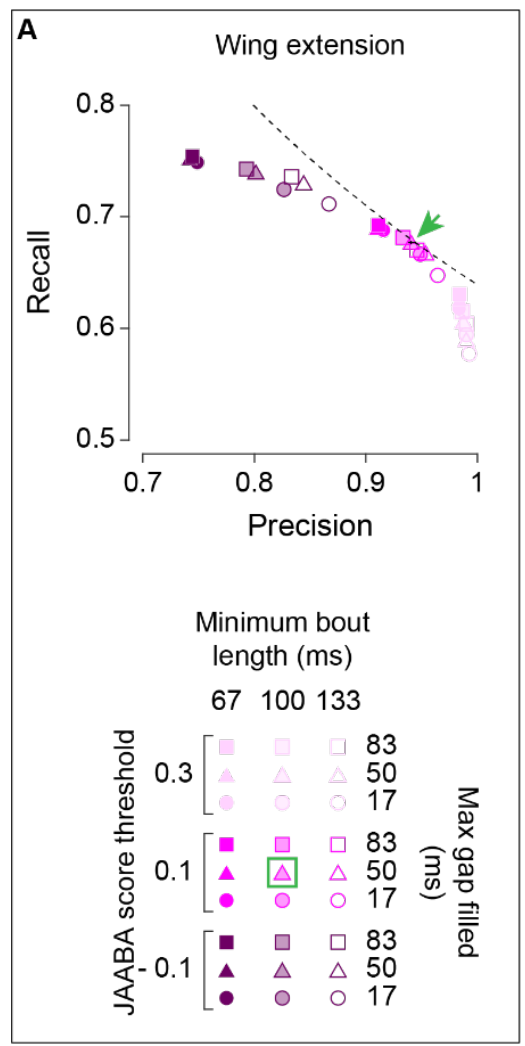

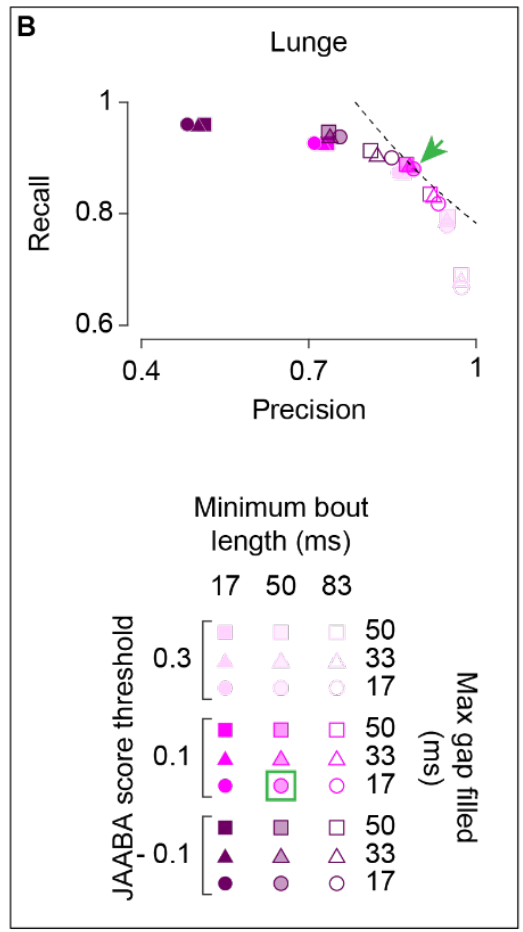

Figure 5. Choice of filter parameters for JAABA bout smoothing.

Recall-precision plot of fully trained classifiers for wing extension $(\mathbf{A})$, lunge $(\mathbf{B})$, and headbutt (C) when average JAABA score threshold, minimum bout length, and maximum gap to be filled are varied as indicated below each plot. Green (arrows and rectangles) indicates the parameter combinations chosen for fully annotated classifiers. Dotted lines are inverse proportion functions that pass the points indicated by green arrows. These combinations have the near-maximum recall $\mathrm{X}$ precision values for each behavior. 
bioRxiv preprint doi: https://doi.org/10.1101/2020.06.16.153130; this version posted June 16, 2020. The copyright holder for this preprint (which was not certified by peer review) is the author/funder, who has granted bioRxiv a license to display the preprint in perpetuity. It is made available under aCC-BY-NC-ND 4.0 International license.

Figure 6

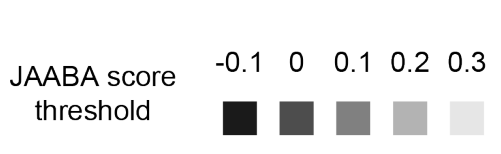

False Bouts False Positive Negative positives detected negatives training bouts training bouts JAABA score a

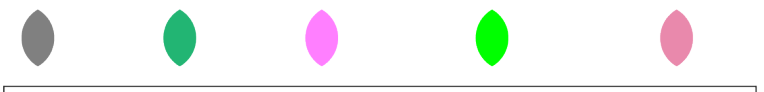

Wing extension
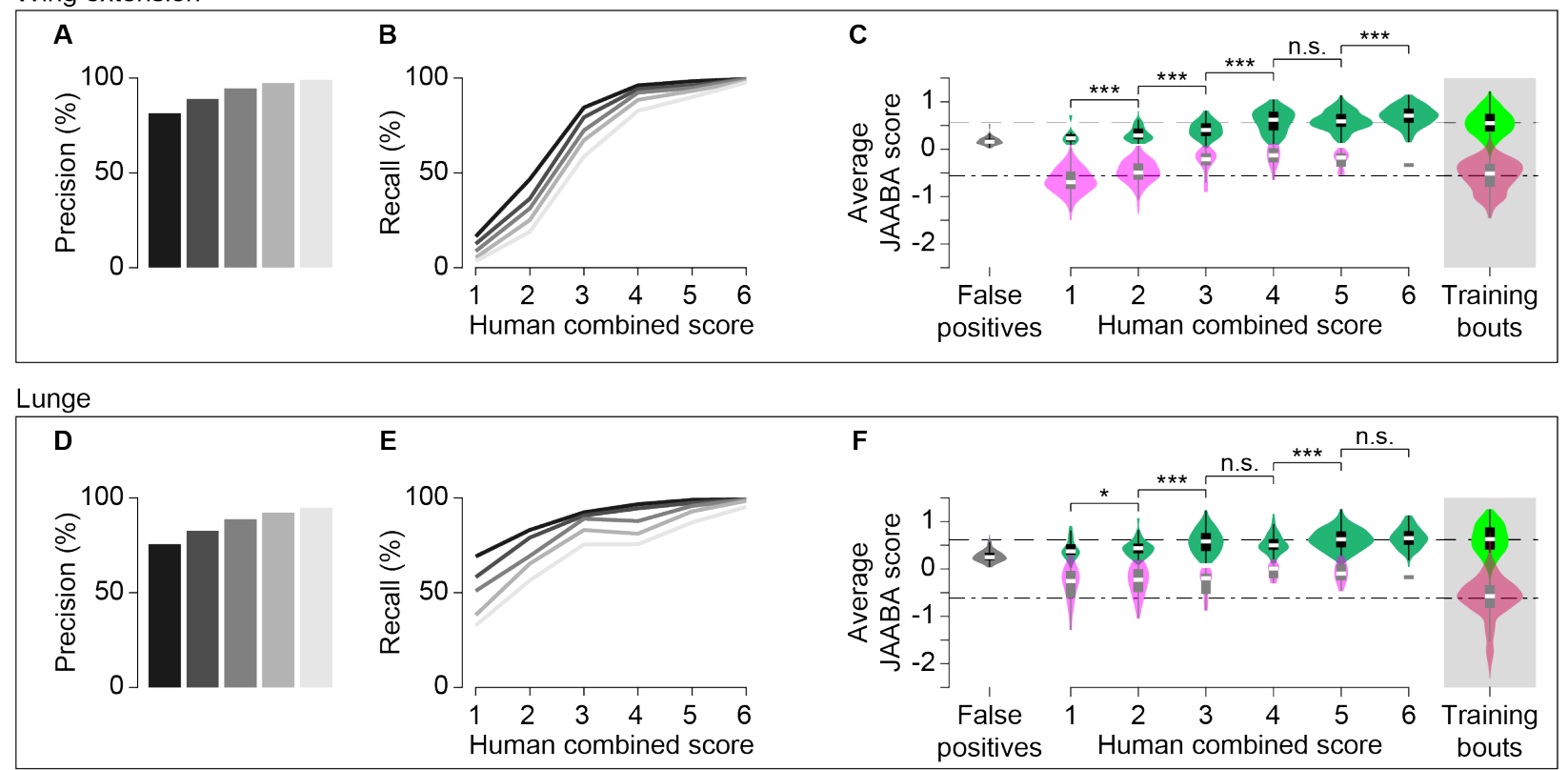

\section{Headbutt}

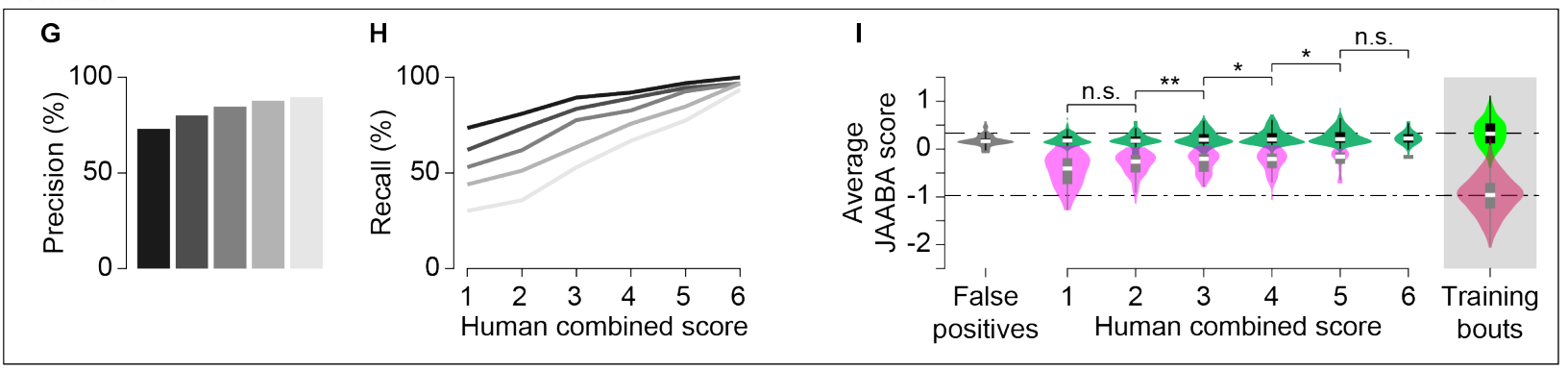

\begin{tabular}{|cccc|}
\hline & Wing & Lunge & Headbutt \\
extension & & \\
Shuffled & & 0 & \\
Observed & $\cdot$ & - & - \\
\hline
\end{tabular}

$\mathbf{J}$

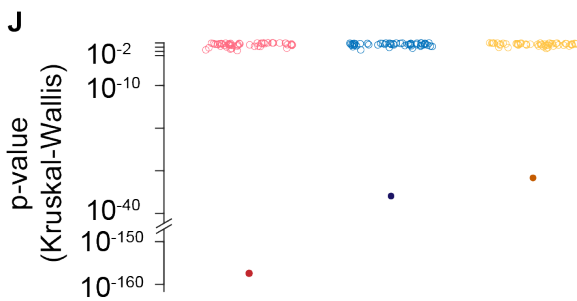

\begin{tabular}{|c|c|c|c|}
\hline & Wing extension & Lunge & Headbutt \\
\hline $\begin{array}{r}\text { Shuffled } \\
\text { (Average) }\end{array}$ & & & \\
\hline Observed & - & 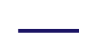 & - \\
\hline
\end{tabular}
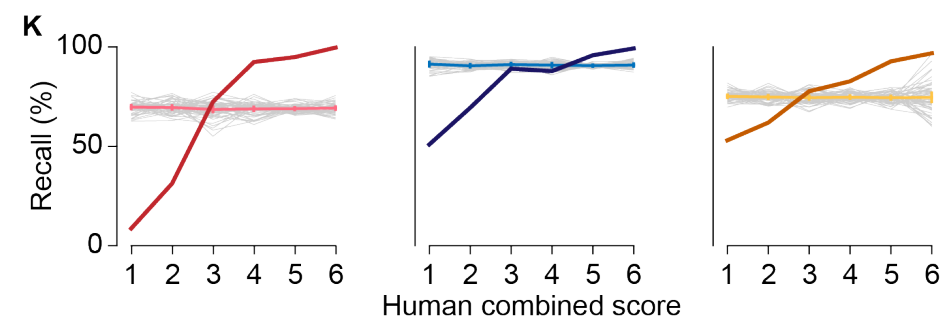
Figure 6. Human confidence and JAABA confidence are correlated.

A-I. Precision (A, D, G) and recall $(\mathbf{B}, \mathbf{E}, \mathbf{H})$ of fully trained classifiers for wing extensions (A, B), lunges $(\mathbf{D}, \mathbf{E})$, and headbutts $(\mathbf{G}, \mathbf{H})$ are shown for varied JAABA score thresholds, as indicated at the top of the figure. For recall, detected bouts were binned according to human combined scores of 1 to 6 . In $\mathbf{C}$ (wing extensions), $\mathbf{F}$ (lunges), and I (headbutts), the distributions of average JAABA scores for true-positive (green), false-negative (pink), and false-positive (gray) bouts are shown as both violin plots (see Materials and Methods for definitions) and box plots. As references, distributions of positive (light green) and negative (crimson) training bouts are shown at right, and the median values for positive and negative training bouts are shown by even and uneven broken lines, respectively. n.s. $p>0.05,{ }^{*} p<0.05,{ }^{* *} p<0.01$, ${ }^{* * *} p<0.001$ by KruskalWallis one-way ANOVA and post-hoc Mann-Whitney U-test. J. Kruskal-Wallis p-value distributions of shuffled (open circles) and observed (filled circles) data sets across human combined scores. K. Recall rates across human combined scores for shuffled and observed data sets at a JAABA score threshold $=0.1$ (observed data sets are replotted from $\mathbf{B}, \mathbf{E}$, and $\mathbf{H}$ ). Average and $95 \%$ confidence intervals for shuffled data are shown in light colors. 
bioRxiv preprint doi: https://doi.org/10.1101/2020.06.16.153130; this version posted June 16, 2020. The copyright holder for this preprint (which was not certified by peer review) is the author/funder, who has granted bioRxiv a license to display the preprint in perpetuity. It is made available under aCC-BY-NC-ND 4.0 International license.

Figure 7

Wing Extention
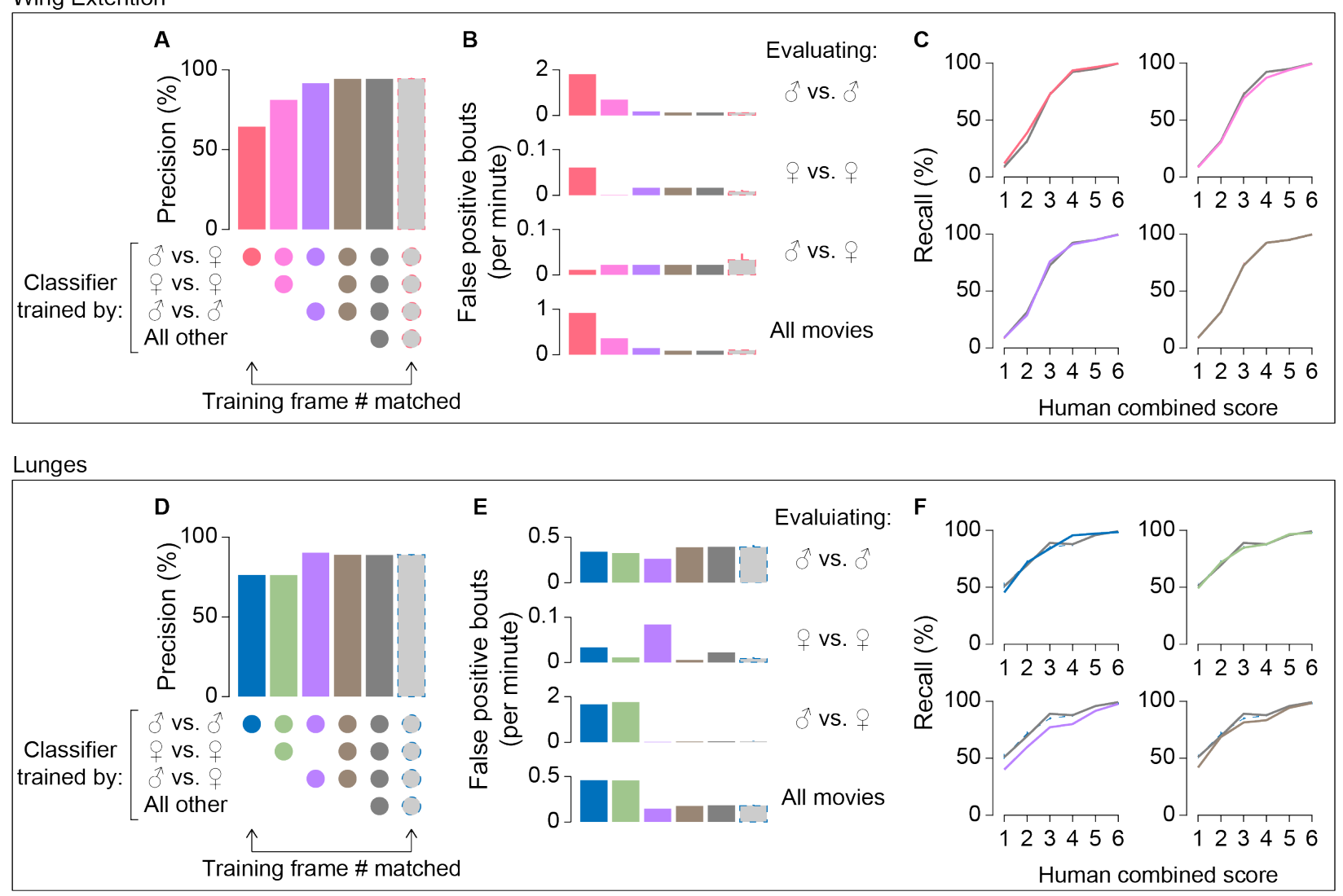

Headbutts

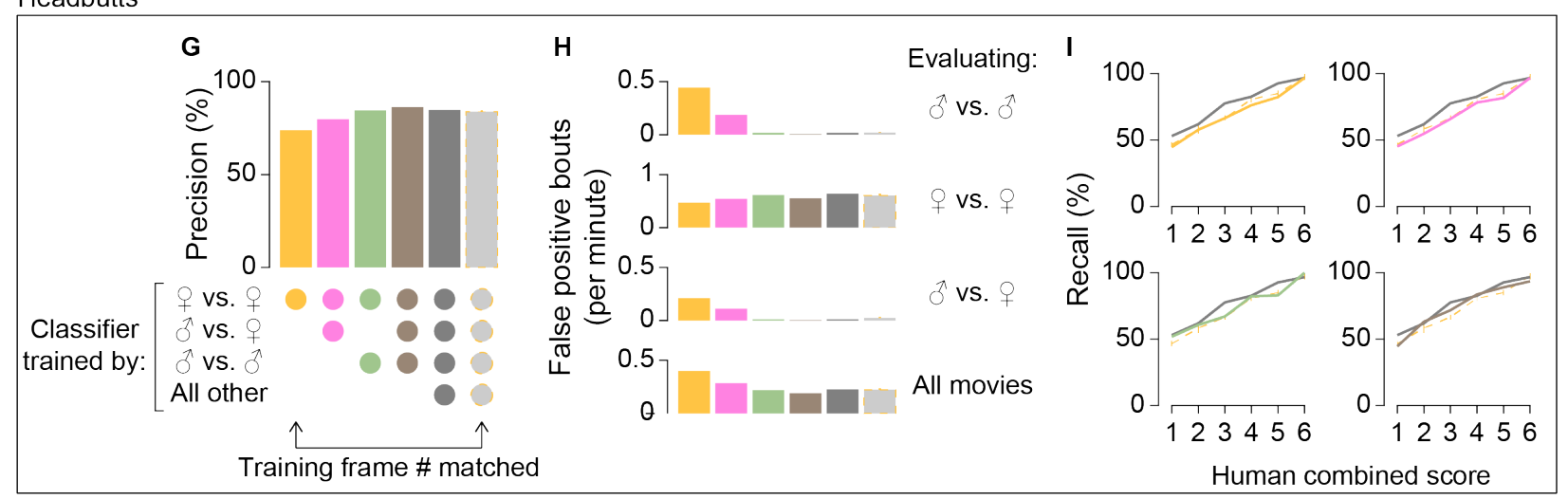


Figure 7. Classifier performance improves as diversity of training frames increases.

Precision (A, D, G), false-positive rates $(\mathbf{B}, \mathbf{E}, \mathbf{H})$, and recall $(\mathbf{C}, \mathbf{F}, \mathbf{I})$ of classifiers for wing extensions (A-C), lunges (D-F), and headbutts $(\mathbf{G}-\mathbf{I})$ are plotted according to the types of training movies used (indicated by the circles below A, D, G). False positive rates are shown separately for the evaluating movie types indicated on the right. "All other" movies include fruitless mutants as indicated in Table S1. Gray bars with broken outlines (A, B, D, E, G, H) and broken lines (C, $\mathbf{F}, \mathbf{I})$ represent the mean and $95 \%$ confidence intervals of the classifiers trained with frames downsampled proportional to the ratio of the training frames from a single type of movie (leftmost bars on $\mathbf{A}, \mathbf{D}, \mathbf{G})$ to the entire number of training frames. Note that the $95 \%$ confidence intervals are generally very small. Also, recall for wing-extension and lunge classifiers with downsampled training frames are very similar to those for fully trained classifiers. Precision and recall for classifiers trained by "all movies" (shown in gray) are replotted from Figure 6. 
Figure 8

Wing Extention
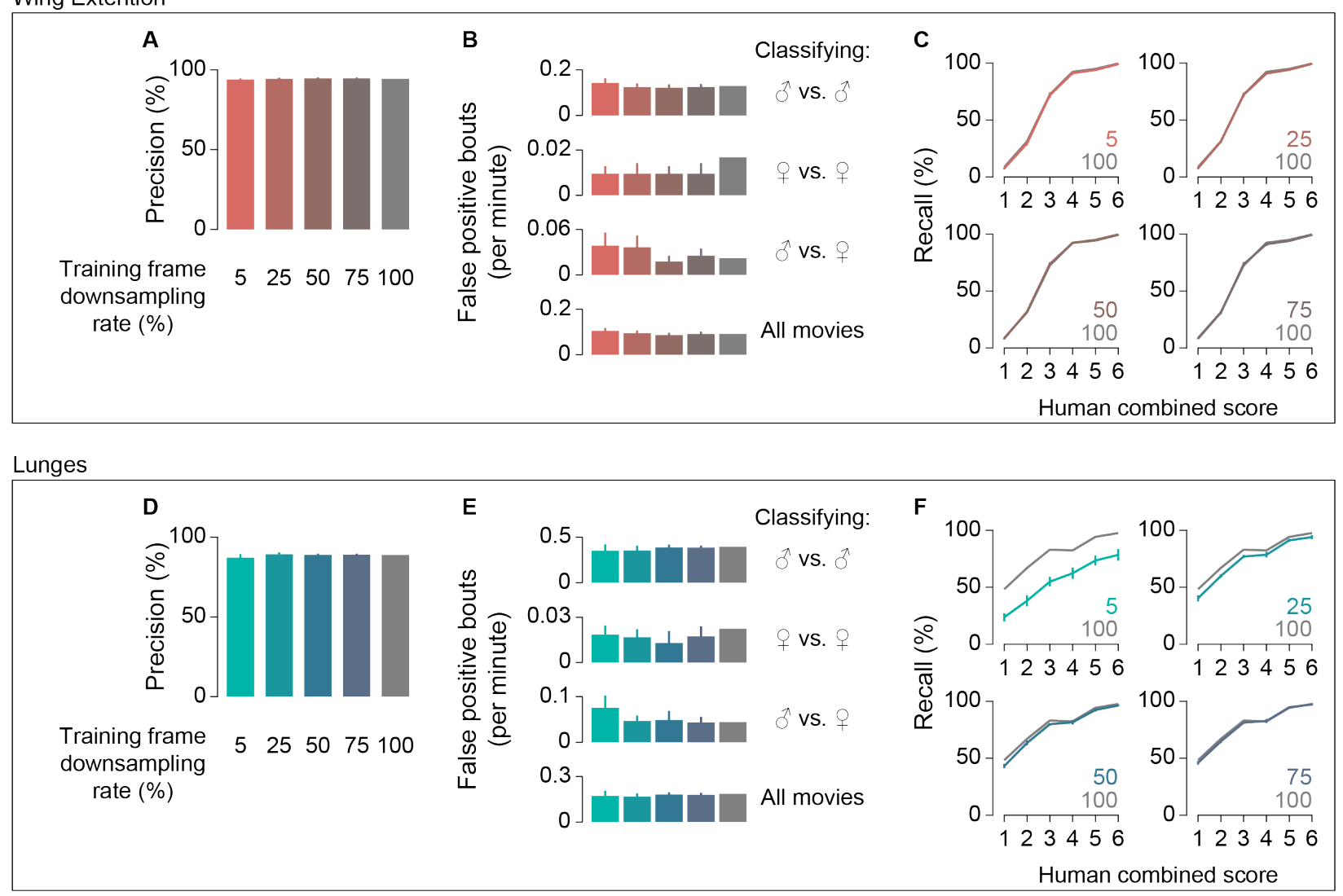

Headbutts

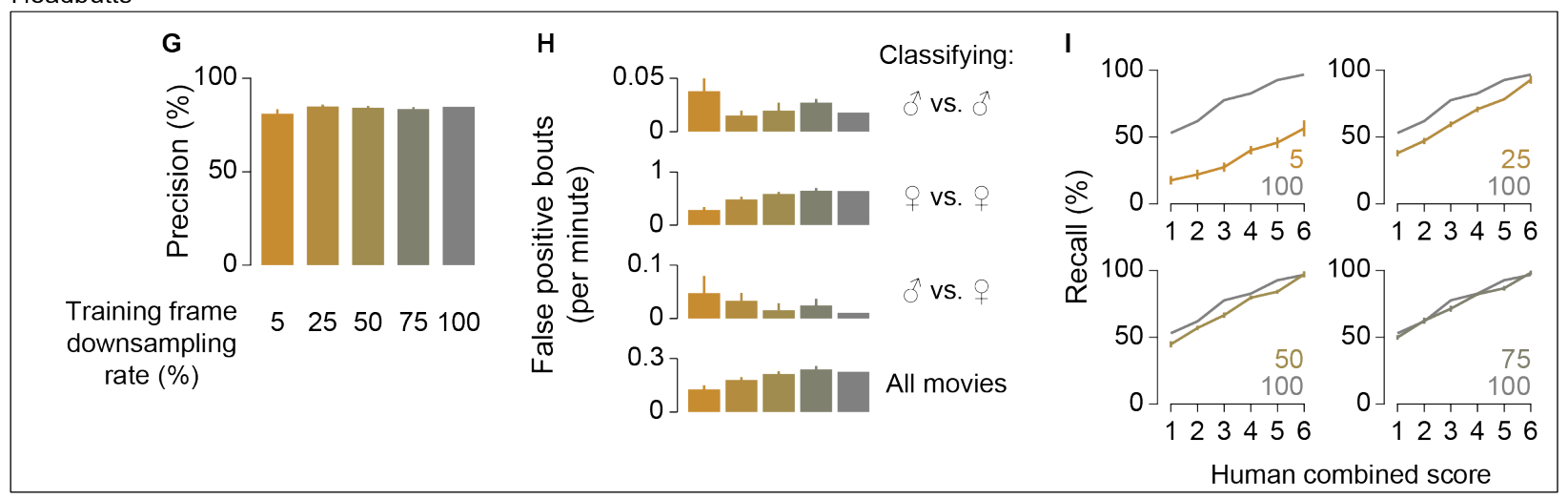

Figure 8. Performance of classifiers with downsampled training frames.

Mean and $95 \%$ confidence intervals (vertical lines) of precision $(\mathbf{A}, \mathbf{D}, \mathbf{G})$, false positive rates (B, $\mathbf{E}, \mathbf{H})$ and recall $(\mathbf{C}, \mathbf{F}, \mathbf{I})$ of classifiers for wing extension $(\mathbf{A}-\mathbf{C})$, lunges (D-F), and headbutt (G-I) trained with downsampled frames (indicated below precision plots and inside recall plots). False positive rates are shown separately for types of movies classified as indicated on the right. Values for classifiers trained at $100 \%$ downsampling rate (all frames used, shown in gray) are replots from Fig. 6. 
Figure 9

Wing Extention
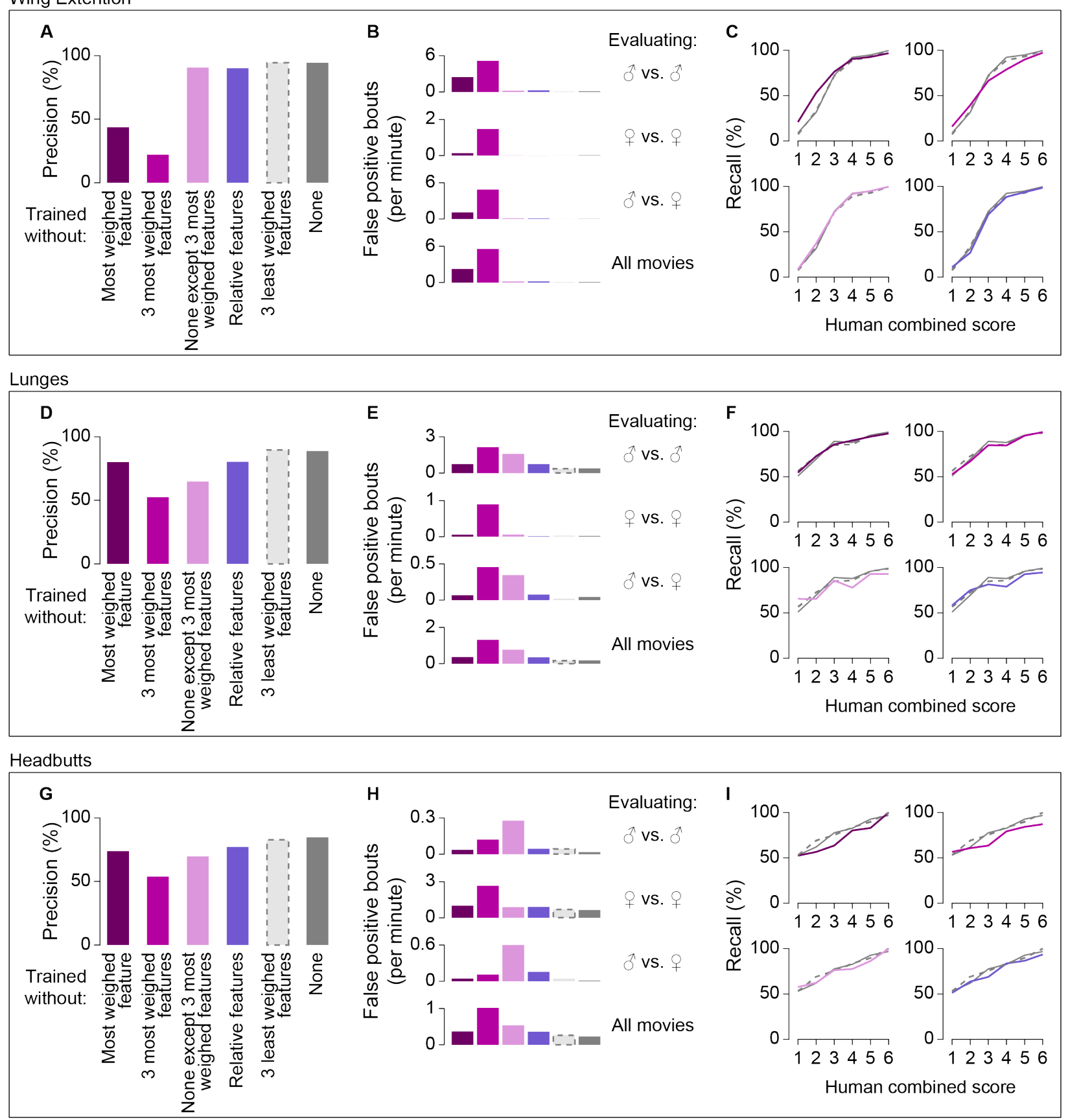

Figure 9. Performance of classifiers change when some rules or features are removed.

Precision (A, D, G), false-positive rates $(\mathbf{B}, \mathbf{E}, \mathbf{H})$, and recall $(\mathbf{C}, \mathbf{F}, \mathbf{I})$ of classifiers for wing extensions (A-C), lunges (D-F), and headbutts (G-I) are plotted according to the features not available for training on JAABA (shown below A, D, G). False-positive rates are shown separately for the evaluating movie types indicated on the right. Precision and recall for classifiers trained with all features (shown in gray) are replotted from Fig. 6. 
Figure 10

Wing extension
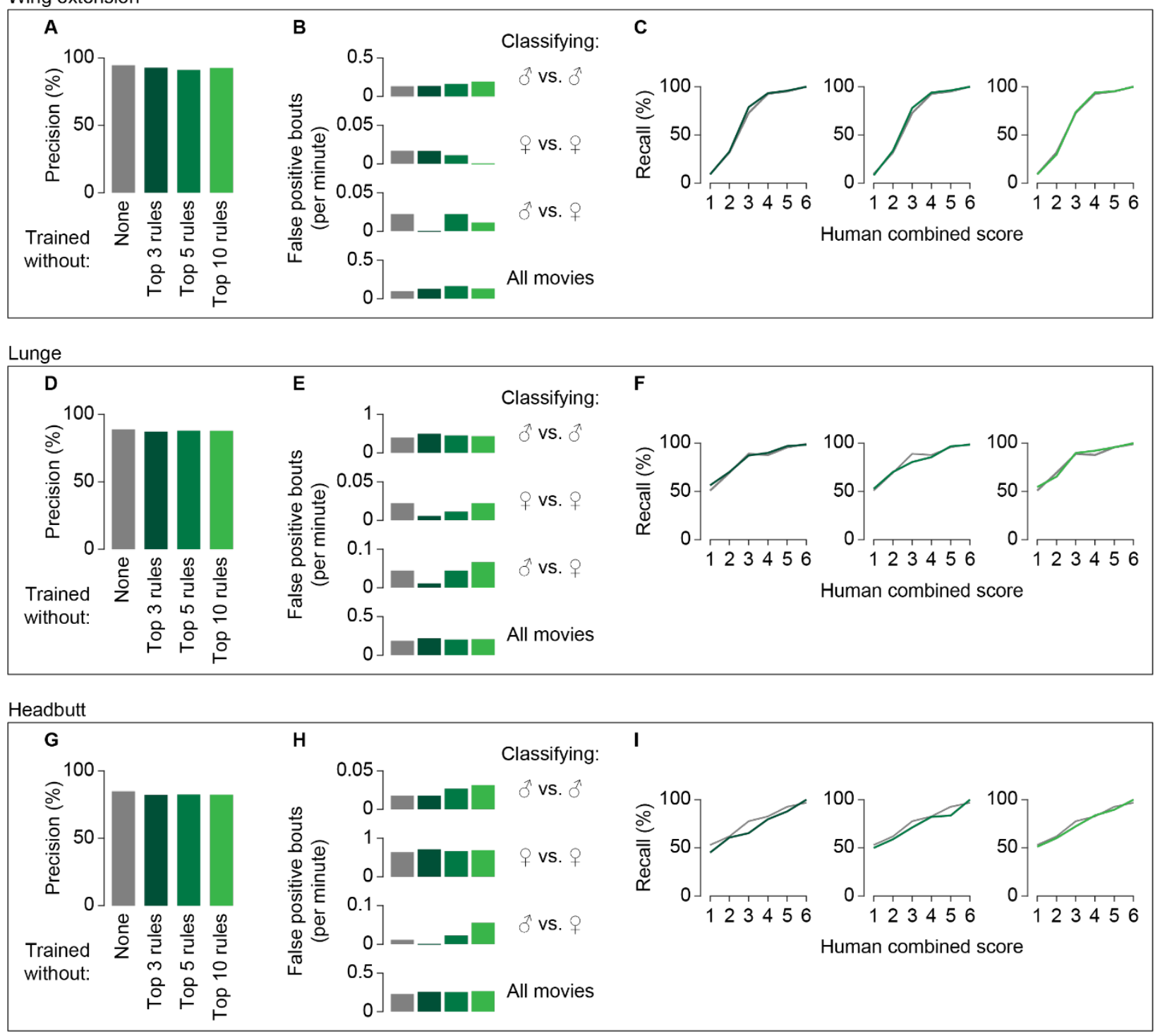

Figure 10. Classifier performs robustly when most weighted rules are removed.

Precision $(\mathbf{A}, \mathbf{D}, \mathbf{G})$, false positive rates $(\mathbf{B}, \mathbf{E}, \mathbf{H})$ and recall $(\mathbf{C}, \mathbf{F}, \mathbf{I})$ of classifiers for wing extension (A-C), lunges (D-F), and headbutt (G-I) trained without the most weighted JAABA rules (indicated below precision plots). False positive rates are shown separately for classifying movie types as indicated on the right. Values for classifiers trained with all rules (shown in gray) are replots from Fig. 6. 
bioRxiv preprint doi: https://doi.org/10.1101/2020.06.16.153130; this version posted June 16, 2020. The copyright holder for this preprint (which was not certified by peer review) is the author/funder, who has granted bioRxiv a license to display the preprint in perpetuity. It is made available under aCC-BY-NC-ND 4.0 International license.

Figure 11

Wing extension (frame-based analyses)

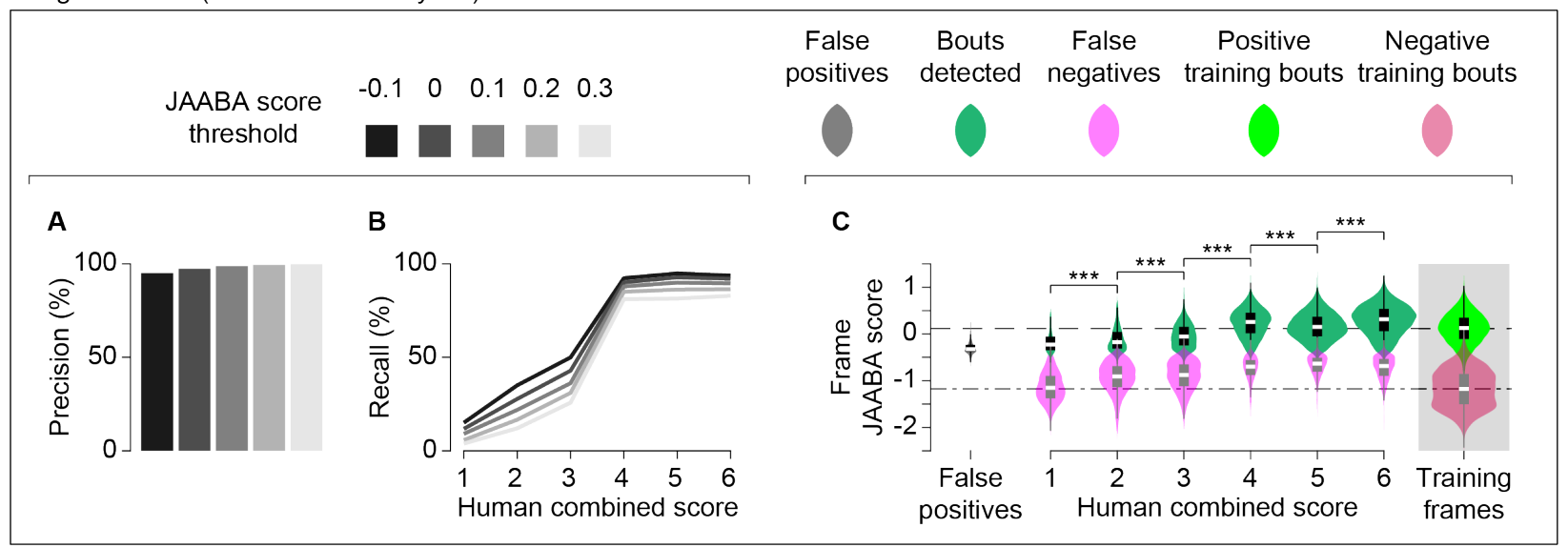
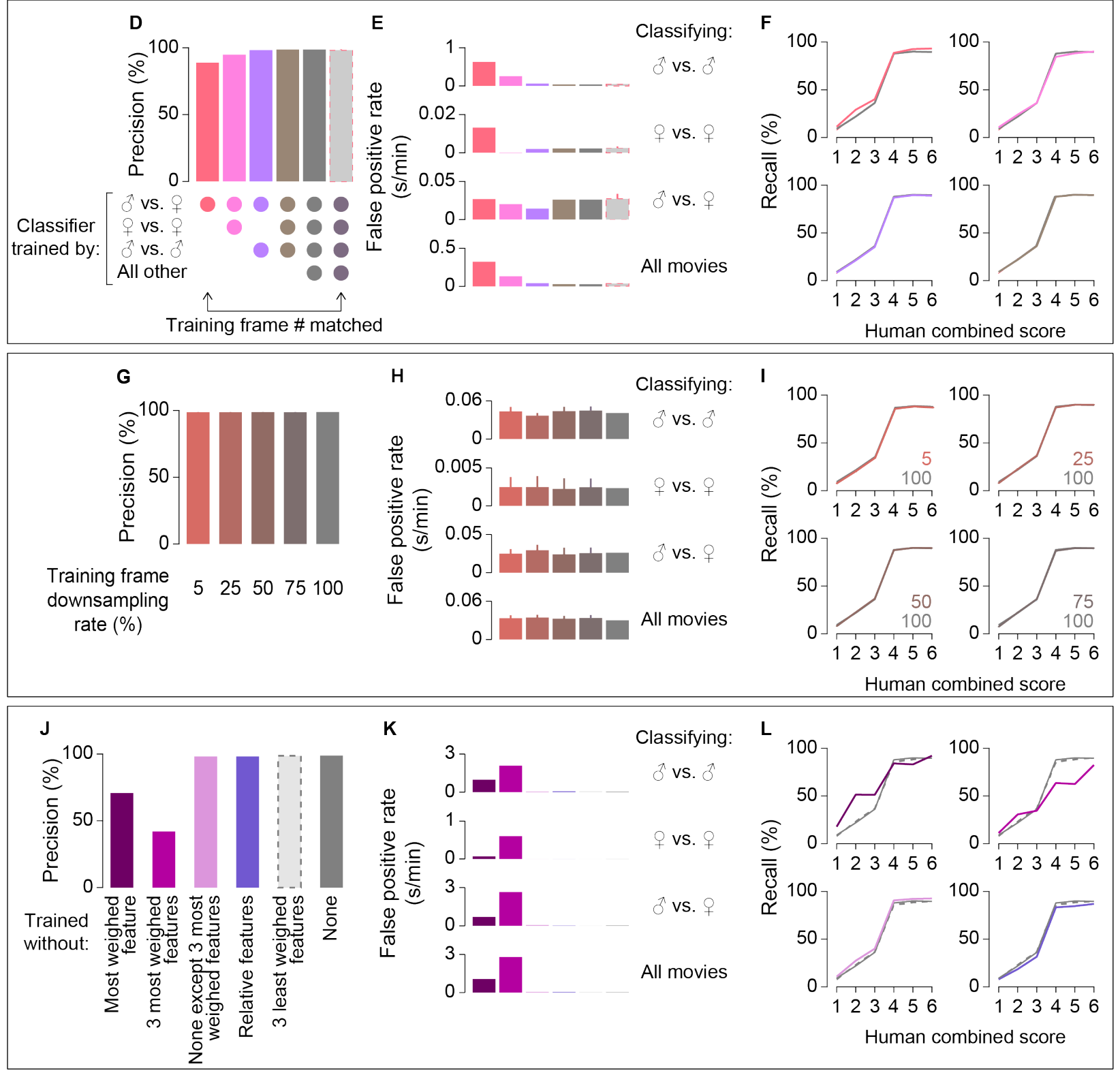
Figure 11. Frame-based analyses of wing extensions classifiers.

A-C. Frame-based plots for precision (A), recall (B), and average JAABA score distribution for each human combined score $(\mathbf{C})$ of the results from the fully trained wing extension classifier (corresponding to Fig. 6A-C). D-F Frame-based plots for precision (D), false positive rates (E), and recall $(\mathbf{F})$ of the results from the wing extension classifiers trained with the specific types of training movies (shown below precision plots) (corresponding to Fig. 7A-C). G-I. Frame-based plots for mean and 95\% confidence intervals (vertical lines) of precision (G), false positive rates $(\mathbf{H})$ and recall $(\mathbf{I})$ of classifiers trained with downsampled frames (rates indicated below precision plots and inside recall plots). False positive rates are shown separately for classifying movie types as indicated on the right. Frame-based plots for precision $(\mathbf{J})$, false positive rates $(\mathbf{K})$, and recall (L) of the results from the wing extension classifiers trained with subsets of features (as indicated below precision plots). (corresponding to Fig. 9A-C). For D-L, dark gray plots represent the value of the fully trained classifier (bars in $\mathbf{D}, \mathbf{G}, \mathbf{J}$ are replots of $A$, and lines in $\mathbf{F}, \mathbf{I}, \mathbf{L}$ are replots of $\mathrm{B}$, respectively, at JAABA score threshold of 0.1 ). 
bioRxiv preprint doi: https://doi.org/10.1101/2020.06.16.153130; this version posted June 16, 2020. The copyright holder for this preprint (which was not certified by peer review) is the author/funder, who has granted bioRxiv a license to display the preprint in perpetuity. It is made available under aCC-BY-NC-ND 4.0 International license.

Table 1: Complete descriptions of the movies annotated by human observers.

- Well \# was counted from left to right, top to bottom.

- A fly that carries $2 X$ chromosomes and frum/fru $4-40$ alleles at the fru locus is called "fruM female". A fly that carries $1 \mathrm{X}$ chromosome and $1 \mathrm{Y}$ chromosome, and fruF/fru ${ }^{4-40}$ alleles at the fru locus, is called "fruF male". These nomenclatures are adapted from $(21,22)$.

\begin{tabular}{|c|c|c|c|c|c|}
\hline Movie names & Length & $\begin{array}{l}\text { Well \# used } \\
\text { for training }\end{array}$ & Genotypes of flies & $\begin{array}{l}\text { Genotypes of } \\
\text { target flies } \\
\text { (wing } \\
\text { clipped), if dif- } \\
\text { ferent }\end{array}$ & $\begin{array}{l}\text { Sex } \\
\text { combi- } \\
\text { nation }\end{array}$ \\
\hline $\begin{array}{l}\text { 033015_NPF3- } \\
\text { CsChrimson- } \\
\text { attP40MG-2.avi }\end{array}$ & $\begin{array}{l}8 \text { min. } 30 \\
\text { sec. }\end{array}$ & $1,2,12$ & $\begin{array}{l}\text { w/Y; 20XUAS- } \\
\text { CsChrimson:mVenus in } \\
\text { attP40/+; NPF-GAL4/+ }\end{array}$ & & o. vs. $\sigma^{\pi}$ \\
\hline $\begin{array}{l}\text { 041815_1_m_g_Otd- } \\
\text { FLPoChrimsonmvenu- } \\
\text { sattP2_f2a20.avi }\end{array}$ & $\begin{array}{l}8 \mathrm{~min} .30 \\
\mathrm{sec} .\end{array}$ & 4,9 & $\begin{array}{l}\text { w, Tk-GAL41/Y; Otd-n/s:FLPo } \\
\text { in attP40/+; } \\
\text { 20XUAS>stop>CsChrimson:m } \\
\text { Venus in attP2/+ }\end{array}$ & & $\delta^{\lambda}$ vs. $\delta^{\lambda}$ \\
\hline $\begin{array}{l}\text { 042015_12_m_g_Otd- } \\
\text { FLPo Chrimsonmvenu- } \\
\text { sattP2_f10a10.avi }\end{array}$ & $\begin{array}{l}8 \text { min. } 30 \\
\text { sec. }\end{array}$ & $3,10,11$ & $\begin{array}{l}\text { w, Tk-GAL41/Y; Otd-nls:FLPo } \\
\text { in attP40/+; } \\
20 X U A S>\text { stop>CsChrimson:m } \\
\text { Venus in attP2/+ }\end{array}$ & & $\delta^{1}$ vs. $0^{1}$ \\
\hline $\begin{array}{l}\text { 042415_4_m_g_Otd- } \\
\text { FLPoChrimson TdTo- } \\
\text { mattP2_CsHeis_F2a2 } \\
0 . \text { avi }\end{array}$ & $\begin{array}{l}8 \mathrm{~min} .30 \\
\mathrm{sec} .\end{array}$ & $1,4,9$ & $\begin{array}{l}\text { w, Tk-GAL41/Y; Otd-n/s:FLPo } \\
\text { in attP40/+; } \\
\text { 20XUAS>myr:TopHAT2>CsCh } \\
\text { rimson:tdTomato in attP2/+ }\end{array}$ & $\begin{array}{l}\text { Wild-type } \\
\text { (Canton-S) } \lesssim\end{array}$ & $\sigma^{\lambda}$ vs. $\sigma^{\lambda}$ \\
\hline 042815_assay1.avi & $30 \mathrm{~min}$. & $6,7,9,12$ & Wild-type (Canton-S) $\hat{O}^{\lambda}$ & & 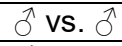 \\
\hline 042815_assay4.avi & $30 \mathrm{~min}$. & $1,5,7$ & Wild-type (Canton-S) $\partial^{\lambda}$ & $\begin{array}{l}\text { Wild-type } \\
\text { (Canton-S) } \text { 우 }\end{array}$ & ôv. $q$ \\
\hline 050815_assay9.avi & $30 \mathrm{~min}$. & 9,11 & Wild-type (Canton-S) & $\begin{array}{l}\text { Wild-type } \\
\text { (Canton-S) } q\end{array}$ & q vs. $q$ \\
\hline 082615_CSMH_SF.avi & $30 \mathrm{~min}$. & $1,3,5,7$ & Wild-type (Canton-S) & $\begin{array}{l}\text { Wild-type } \\
\text { (Canton-S) } q\end{array}$ & q vs. $q$ \\
\hline 100815_4.avi & $10 \mathrm{~min}$. & $1,4,10,12$ & $\begin{array}{l}\text { w, Tk-GAL41/w; Otd-nls:FLPo } \\
\text { in attP40/+; } \\
20 X U A S>\text { myr:TopHAT2>CsCh } \\
\text { rimson:tdTomato in attP2, fru }{ }^{4-} \\
40 / \mathrm{fru}^{M}\end{array}$ & $\begin{array}{l}+/ Y ;+; \\
\text { fru } / \text { fru }\end{array}$ & $\begin{array}{l}\text { Other } \\
\text { (fruM } \text { + } \\
\text { vs fruF } \\
\delta^{\pi} \text { ) }\end{array}$ \\
\hline $\begin{array}{l}\text { 2016_02_15_CsMH_M } \\
\text { SH2.avi }\end{array}$ & $5 \mathrm{~min}$. & 1 & Wild-type (Canton-S) $\hat{O}^{\wedge}$ & $\begin{array}{l}\text { Wild-type } \\
\text { (Canton-S) }{ }^{\wedge}\end{array}$ & $\hat{\sigma}$ vs. $\sigma^{\pi}$ \\
\hline $\begin{array}{l}\text { 2016_02_15_CsMH_M } \\
\text { SH3.avi }\end{array}$ & $5 \mathrm{~min}$. & 2 & Wild-type (Canton-S) $\widehat{\delta}$ & $\begin{array}{l}\text { Wild-type } \\
\text { (Canton-S) }{ }^{\lambda}\end{array}$ & $\hat{0}$ vs. $\sigma^{\lambda}$ \\
\hline
\end{tabular}


bioRxiv preprint doi: https://doi.org/10.1101/2020.06.16.153130; this version posted June 16, 2020. The copyright holder for this preprint (which was not certified by peer review) is the author/funder, who has granted bioRxiv a license to display the preprint in perpetuity. It is made available under aCC-BY-NC-ND 4.0 International license.

Table 2: Annotations of false positives by the wing extension classifier:

- Green categories indicate motions that are similar to actual behaviors.

\begin{tabular}{|c|c|c|c|c|c|}
\hline \multirow[t]{2}{*}{ Types of motions } & \multicolumn{5}{|c|}{ False positive counts } \\
\hline & $\hat{0}$ vs. $\hat{O}^{\lambda}$ & ô vs. ㅇ & q vs. $q$ & $\begin{array}{l}\text { Other (fruM } \\
+ \text { vs fruF }{ }^{\lambda} \text { ) }\end{array}$ & Total $(\%)$ \\
\hline Actual wing extensions & 5 & 0 & 0 & 6 & $11(22.4)$ \\
\hline Wing glooming & 6 & 0 & 2 & 4 & $12(24.5)$ \\
\hline Incomplete wing closure & 8 & 0 & 1 & 3 & $12(24.5)$ \\
\hline $\begin{array}{l}\text { Wing tracking error (including ac- } \\
\text { tions when flies are on or near the } \\
\text { wall) }\end{array}$ & 7 & 1 & 0 & 1 & $9(18.4)$ \\
\hline Wing threats & 2 & 0 & 0 & 1 & $3(6.1)$ \\
\hline Others & 1 & 1 & 0 & 0 & $2(4.1)$ \\
\hline
\end{tabular}

Table 3: Annotations of false positives by the lunge classifier:

\begin{tabular}{|c|c|c|c|c|c|}
\hline \multirow[t]{2}{*}{ Types of motions } & \multicolumn{5}{|c|}{ False positive counts } \\
\hline & $\hat{\sigma}$ vs. $\hat{\jmath}$ & ô vs. + ? & $q$ vs. + & $\begin{array}{l}\text { Other (fruM } \\
+ \text { vs fruF }{ }^{\lambda} \text { ) }\end{array}$ & Total $(\%)$ \\
\hline Actual or incomplete lunges & 24 & 0 & 0 & 1 & $25(25.3)$ \\
\hline Lunge-like actions during tussling & 20 & 0 & 0 & 0 & $20(20.2)$ \\
\hline $\begin{array}{l}\text { Actions when flies are on or near } \\
\text { the wall (ex. falling from the wall) }\end{array}$ & 17 & 1 & & 2 & $20(20.2)$ \\
\hline Receiving lunge or headbutt & 13 & 0 & 2 & 0 & $15(15.2)$ \\
\hline $\begin{array}{l}\text { Fast autonomous motions (jump- } \\
\text { ing, rolling on floor, etc.) }\end{array}$ & 14 & 1 & 0 & 0 & $15(15.2)$ \\
\hline Copulation attempt & 0 & 2 & 0 & 0 & $2(2.0)$ \\
\hline $\begin{array}{l}\text { Reaction to other fly's fast autono- } \\
\text { mous motions }\end{array}$ & 0 & 0 & 2 & 0 & $2(2.0)$ \\
\hline
\end{tabular}

Table 4: Annotations of false positives by the headbutt classifier:

\begin{tabular}{|c|c|c|c|c|c|}
\hline \multirow[t]{2}{*}{ Types of motions } & \multicolumn{5}{|c|}{ False positive counts } \\
\hline & $0^{1}$ vs. $\hat{o}^{1}$ & ôvs. ㅇ & q vs. + & $\begin{array}{l}\text { Other (fruM } \\
+ \text { vs fruF } \delta^{\lambda} \text { ) }\end{array}$ & Total $(\%)$ \\
\hline Actual headbutt & 0 & 1 & 7 & 1 & $8(6.6)$ \\
\hline Jerking toward the other fly & 1 & 0 & 23 & 0 & $24(19.8)$ \\
\hline $\begin{array}{l}\text { Walking toward the other fly while } \\
\text { extending a leg ('reaching') }\end{array}$ & 0 & 0 & 24 & 0 & $24(19.8)$ \\
\hline Receiving lunge or headbutt & 0 & 0 & 22 & 0 & $22(18.2)$ \\
\hline $\begin{array}{l}\text { Fast autonomous motions (jump- } \\
\text { ing, rolling on floor, etc.) }\end{array}$ & 0 & 0 & 21 & 0 & $21(17.4)$ \\
\hline $\begin{array}{l}\text { Reaction to other fly's fast autono- } \\
\text { mous motions }\end{array}$ & 1 & 0 & 7 & 1 & $9(7.4)$ \\
\hline Pushing the other fly & 0 & 0 & 8 & 0 & $8(6.6)$ \\
\hline $\begin{array}{l}\text { Actions when flies are on or near } \\
\text { the wall (ex. falling from the wall) }\end{array}$ & 1 & 0 & 2 & 0 & $3(2.5)$ \\
\hline Lunge & 0 & 0 & 1 & 0 & $1(0.8)$ \\
\hline $\begin{array}{l}\text { Wing threat with fast motion to- } \\
\text { ward the other fly ('charge') }\end{array}$ & 1 & 0 & 0 & 0 & $1(0.8)$ \\
\hline
\end{tabular}


bioRxiv preprint doi: https://doi.org/10.1101/2020 06.16.153130; this version posted June 16, 2020. The copyright holder for this preprint (which was not certified by peer review) is the author/funder, who has granted bioRxiv a license to display the preprint in perpetuity. It is made available under aCC-BY-NC-ND 4.0 International license.

Table 5: Weights given to features in each classifier (ascending order):

- Features in brown indicate relative features

\begin{tabular}{|c|c|c|c|c|c|}
\hline \multicolumn{2}{|c|}{ Wing extension classifier } & \multicolumn{2}{|l|}{ Lunge classifier } & \multicolumn{2}{|l|}{ Headbutt classifier } \\
\hline Feature & Weight & Feature & Weight & Feature & Weight \\
\hline log max wing ang & 5.678 & norm axis ratio & 3.416 & $\overline{l o g}$ vel & 3.569 \\
\hline facing angle & 2.690 & dist to other & 1.948 & dist to other & 2.298 \\
\hline $\begin{array}{l}\text { norm_mean_wing_le } \\
\text { ngth }\end{array}$ & 2.249 & $\begin{array}{l}\text { norm_mean_wing_le } \\
\text { ngth }\end{array}$ & 1.870 & facing_angle & 1.230 \\
\hline log_min_wing_ang & 1.152 & facing_angle & 1.616 & log_ang_vel & 1.165 \\
\hline dist_to_wall & 1.118 & log_max_wing_ang & 0.937 & dist_to_wall & 1.119 \\
\hline norm_axis_ratio & 0.867 & leg_dist & 0.899 & leg_dist & 1.049 \\
\hline log_fg_body_ratio & 0.661 & dist_to_wall & 0.697 & $\begin{array}{l}\text { norm_mean_wing_le } \\
\text { ngth }\end{array}$ & 0.960 \\
\hline leg_dist & 0.650 & log_vel & 0.625 & log_max_wing_ang & 0.853 \\
\hline log_vel & 0.498 & angle_between & 0.539 & log_fg_body_ratio & 0.842 \\
\hline dist to other & 0.342 & log_min_wing_ang & 0.459 & norm_axis_ratio & 0.816 \\
\hline log_ang_vel & 0.228 & log_fg_body_ratio & 0.411 & norm_contrast & 0.774 \\
\hline norm_contrast & 0.133 & norm_contrast & 0.406 & log_min_wing_ang & 0.380 \\
\hline angle between & 0.110 & log_ang_vel & 0.246 & angle_between & 0.334 \\
\hline
\end{tabular}

Supplementary Tables and Supplementary Data Files are available in .xlsx format upon request. 